Joint Detection and Tracking of Unresolved Targets with a Monopulse Radar Using a Particle Filter 


\title{
JOINT DETECTION AND TRACKING OF UNRESOLVED TARGETS WITH A MONOPULSE RADAR USING A PARTICLE FILTER
}

\author{
By \\ N. NANDAKUMARAN, B.Sc.Eng. \\ University of Peradeniya, Peradeniya, Sri Lanka, 2001
}

\author{
A Thesis \\ Submitted to the School of Graduate Studies \\ in Partial Fulfilment of the Requirements \\ for the Degree \\ Master of Applied Science
}

McMaster University

September 2005 
MASTER OF APPLIED SCIENCE (2005)

MCMASTER UNIVERSITY

(Electrical and Computer Engineering)

Hamilton, Ontario, Canada

TITLE:

Joint Detection and Tracking of

Unresolved Targets with a Monopulse Radar

Using a Particle Filter

AUTHOR:

N. Nandakumaran

B.Sc.Eng.

University of Peradeniya

Peradeniya, Sri Lanka, 2001

SUPERVISOR:

Dr. T. Kirubarajan

NUMBER OF PAGES: viii, 62 
To my parents, sister and brothers 


\section{Abstract}

Detection and estimation of multiple unresolved targets with a monopulse radar is a challenging problem. For ideal single bin processing, it was shown in the literature that at most two unresolved targets can be extracted from the complex matched filter output signal. In this thesis, a new algorithm is developed to jointly detect and track more than two targets from a single detection. This method involves the use of tracking data in the detection process. For this purpose, target states are transformed into the detection parameter space, which involves high nonlinearity. In order to handle this, the sequential Monte Carlo (SMC) method, which has proven to be effective in nonlinear non-Gaussian estimation problems, is used as the basis of the closed loop system for tracking multiple unresolved targets. In addition to the standard SMC steps, the detection parameters corresponding to the predicted particles are evaluated using the nonlinear monopulse radar beam model. This in turn enables the evaluation of the likelihood of the monopulse signal given tracking data. Hypothesis testing is then used to find the correct detection event. The particles are updated and resampled according to the hypothesis that has the highest likelihood (score). A simulated amplitude comparison monopulse radar is used to generate the data and to validate the extraction and tracking of more than two unresolved targets. 


\section{Acknowledgements}

Praise be to God for helping me finish this thesis and in all aspects of my life. I wish to express my deepest gratitude to my supervisor Dr. T. Kirubarajan for offering me an opportunity to be a graduate student, and his guidance and inspiration throughout the entire research for this thesis. His patience with students and willingness to always provide extra help are greatly appreciated. I would like to thank Dr. Abhijit Sinha for his guidance and kind suggestions throughout my work.

My appreciation also goes to all the instructors in the ECE Department who taught me various courses. What they have taught me in the class has greatly helped me understand the research papers. Department staff members, especially Cheryl Gies and Helen Jachna, are also gratefully acknowledged for their constant administrative help and support. I thank Terry Greenlay and Cosmin Coriou assisting in resolving computer related issues.

I am thankful to my fellow students in the ETF Laboratory for many thought-provoking and stimulating discussions. All above, I would like to acknowledge the immeasurable contribution of my parents in teaching me the value of education. I am deeply grateful to them, for having sacrificed a lot to bring my siblings and I to where we are now. Finally, I wish to thank my cousin and his family, my roommates and my friends. Their continuous encouragement has been extraordinary and wonderful. 


\section{Contents}

1 Introduction $\quad 1$

1.1 Unresolve Targets with a Monopulse Radar . . . . . . . . . . . . . . . . . . 1

1.2 Contribution of the Thesis . . . . . . . . . . . . . . . . . 2

1.3 Organization of the Thesis . . . . . . . . . . . . . . . 4

1.4 Related Publications . . . . . . . . . . . . . . . . . . 4

2 Monopulse Radar $\quad 5$

2.1 Amplitude Comparison Monopulse Radar . . . . . . . . . . . . . . . . . . . . 6

2.2 Monopulse Radar Signal Processing . . . . . . . . . . . . . . . . . . . . 7

2.3 Monopulse Signals with $N$ Unresolved Targets . . . . . . . . . . . . . 8

2.4 Distribution of Monopulse Signals . . . . . . . . . . . . . . . . . . . 12

3 Detection and Tracking $\quad 14$

3.1 Detection Theory . . . . . . . . . . . . . . . . . 15

3.1.1 Binary Hypothesis Test . . . . . . . . . . . . . . 15

3.1 .2 Multiple Hypotheses Test . . . . . . . . . . . . . . . 17

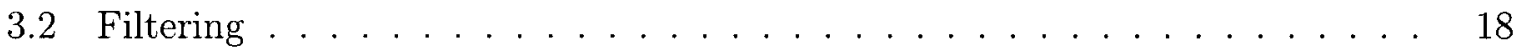

3.2 .1 Kalman Filter . . . . . . . . . . ........ 18

3.2 .2 Grid Based Filter . . . . . . . . . . . . . . . . . 20 
3.2 .3 Extended Kalman Filter . . . . . . . . . . . . . . . . . . . . 22

3.2 .4 Particle Filter . . . . . . . . . . . . . . . . . . 23

3.3 Data Association . . . . . . . . . . . . . . . . . . . 25

3.3 .1 Gating . . . . . . . . . . . . . . . 26

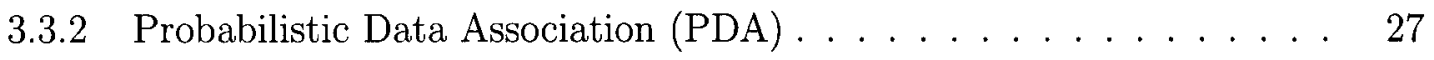

3.3.3 Joint Probabilistic Data Association (JPDA) . . . . . . . . . . . 29

4 Joint Detection And Tracking 32

4.1 Single Recursion of the Algorithm . . . . . . . . . . . . . . . 32

4.2 Block Diagram for Recursion . . . . . . . . . . . . . . . . . 37

5 Posterior Covariance of Target State 38

5.1 Background . . . . . . . . . . . . . . . . . . . . . 38

5.2 Posterior Cramér-Rao Bounds for Target State Estimation . . . . . . . . . 39

6 Simulation Studies $\quad 45$

$\begin{array}{lll}7 & \text { SUMMARY } & \mathbf{5 7}\end{array}$

7.1 Conclusions . . . . . . . . . . . . . . . . . 57

7.2 Recommendations for Future Work . . . . . . . . . . . . . 58 


\section{List of Figures}

2.1 Monopulse radar beams $\ldots \ldots \ldots \ldots$

2.2 Monopulse radar signal processing for a single angular dimension . . . . . . . 7

3.3 A typical tracking system $\ldots \ldots \ldots \ldots$

4.4 Joint detection and tracking $\ldots \ldots \ldots \ldots \ldots \ldots$

6.5 Target trajectories of the first scenario $\ldots \ldots \ldots \ldots$

6.6 Tracks from 50 Monte Carlo runs (average tracks) $\ldots \ldots \ldots$. . . . . . . . 49

6.7 Tracks from 50 Monte Carlo runs (overlayed) . . . . . . . . . . . . 50

6.8 Average position RMSE . . . . . . . . . . . . . . . . . . 51

6.9 Target trajectories of the second scenario set with three targets $\ldots \ldots \ldots 3$

6.10 Average position RMSE and posterior position standard deviation lower bound for a single target . . . . . . . . . . . . . . . . 54

6.11 Average position RMSE and posterior position standard deviation lower bound for two unresolved targets . . . . . . . . . . . . . . . 55

6.12 Average position RMSE and posterior position standard deviation lower bound for three unresolved targets $\ldots \ldots \ldots \ldots \ldots$ 


\section{Chapter 1}

\section{Introduction}

\subsection{Unresolve Targets with a Monopulse Radar}

The simultaneous lobing technique, better known as Monopulse processing, is widely used in radars to measure the angle of arrival (AOA) of a target with sub-beam accuracy. In amplitude comparison monopulse radar, two squinted beams (per angular coordinate) are used to receive two diverse returns of the target. The comparison of the in-phase difference signal and the in-phase sum signal yields the Direction Of Arrival (DOA) of the target. This method is more preferred over other conventional methods like maximum likelihood estimator and moment matching for its simplicity with estimation accuracy close to the Cramer-Rao lower bound (CRLB) at moderate or high signal-to-noise ratios (SNRs). However, it fails when multiple targets fall into the same resolution cell. In that case, the DOA estimate indicated by the in-phase monopulse ratio can wander far beyond the angular separation of the targets [22]. These merged measurements often result in high errors in the ensuing data association and track filtering algorithms. 
Various methods have been proposed in the literature to extract unresolved targets from radar returns. Some involve special antenna configuration $[13,17,18]$ or array signal processing (beamforming, interference nulling or high-resolution direction finding) $[10,15]$. Others involve the complete utilization of the standard monopulse system, which is the focus of this thesis $[5-7,22-24,29]$. In [22], the complex ratio was used to estimate the DOAs of two fixed amplitude targets with known Relative Radar Cross Section (RRCS) by applying the method of moments. This method has limited applicability due to RRCS fluctuations in real problems. Statistical description of complex monopulse parameters were explicitly derived in [6]. With this description, Neymen-Pearson hypothesis testing was applied to detect target multiplicity in [5]. In [7], it was further developed to estimate DOAs of two unresolved Rayleigh (Swelling I) targets with known or estimated RRCS. For two unresolved Rayleigh targets, the maximum likelihood (ML) angle extractor using numerical method is proposed in [23]. It was proven to be effective for sea-surface multipath monopulse radar signals [24]. But a closed-form solution for this ML extractor was derived in [29]. In [31,32], a more realistic case, where targets' spillover in the adjacent bins was taken into account, was considered. Using the identifiability principle, it was inferred that up to five targets can be extracted using joint bin processing of two adjacent matched filter samples. This in turn implies that using ideal bin processing, as in $[7,23,29]$, at most two targets can be pulled out.

\subsection{Contribution of the Thesis}

All existing methods consider the detection and tracking of the unresolved targets separately. In this thesis, they are performed jointly so that tracking information can be utilized in detection and vice versa. This requires the transformation of target state into the detection 
parameter space, which involves high nonlinearity. The sequential Monte Carlo (SMC) method $[2,8,20]$, also known as the particle filter, which has proven to be effective for nonlinear non-Gaussian estimation problems, makes it possible. With SMC as the basis, a new algorithm is developed to jointly detect and track unresolved targets using an amplitude comparison monopulse radar with ideal single bin processing. It enables us to jointly detect and track more than two targets from a single cell.

In addition to the standard SMC steps, the directions of arrival (DOAs) corresponding to the predicted particle states are evaluated using the nonlinear monopulse radar beam model. All hypotheses corresponding to ideal bin processing (i.e., all possible combinations of targets being in different detected bins) are constructed. The likelihood of monopulse signals given the DOAs of the particles of the different targets and given a particular hypothesis is computed based on the distribution of the monopulse signal. This likelihood is also the joint weight of the particles. The likelihood of a hypothesis is shown to be the sum of the corresponding joint weights. The hypothesis with the highest score is then selected and the corresponding particle sets, which are updated with the marginal of the above joint weights, are taken to be the correct representation of posterior of the target states. That completes a recursion of the SMC. Since detection and tracking are performed jointly, estimation of the DOA of each target is no longer needed. Hence DOAs are not explicitly estimated. In addition, the hypothesis testing of detection events incorporates the data association process as well. The recursive Riccati-like formula for the Fisher Information matrix for nonlinear state estimation from [27] is used to derive the posterior covariance of the target state, which enables us to assess the proposed algorithm. 


\subsection{Organization of the Thesis}

This thesis is organized as follows. In Chapter 2, a brief introduction to monopulse radar systems is given. In addition, it gives the monopulse signal distribution that is used later. Chapter 3 reviews detection theory and the track filtering. In Chapter 4, the proposed algorithm is presented. In Chapter 5, the posterior covariance matrix of target state is derived. The simulation results are given in Chapter 6. Finally, Chapter 7 gives conclusions and suggestions for future work.

\subsection{Related Publications}

1. N. Nandakumaran, A. Sinha and T. Kirubarajan, "Joint Detection and Tracking of Unresolved Targets with a Monopulse Radar Using a Particle Filter", Proceedings of the SPIE Conference of Signal and Data Processing of Small Targets, San Diego, CA, Aug. 2005.

2. N. Nandakumaran, A. Sinha and T. Kirubarajan, "Joint Detection and Tracking of Unresolved Targets with a Monopulse Radar Using a Particle Filter", To be submitted to IEEE Transactions on Aerospace and Electronic Systems. 


\section{Chapter 2}

\section{Monopulse Radar}

This thesis is concerned with handling unresolved targets using a monopulse radar. The goal of this chapter is to provide the necessary background on monopulse radar systems. In addition, this chapter provides the distribution of monopulse signals that is used in later chapters. Several references that explain the monopulse system are also listed in the bibliography [6], [19], [22], [25], [30].

Monopulse processing is the simultaneous lobing technique where the angular measurement is made on the basis of one pulse rather than many pulses. It has significant advantages with respect to target echo fluctuation over other lobing methods, for example, the conical-scan and sequential-lobing techniques, which are severely affected by pulse-to-pulse amplitude fluctuation. There are two kinds of monopulse techniques, namely, amplitude comparison monopulse and phase comparison monopulse. In this thesis, we focus on tracking unresolved targets with amplitude comparison monopulse radar. 


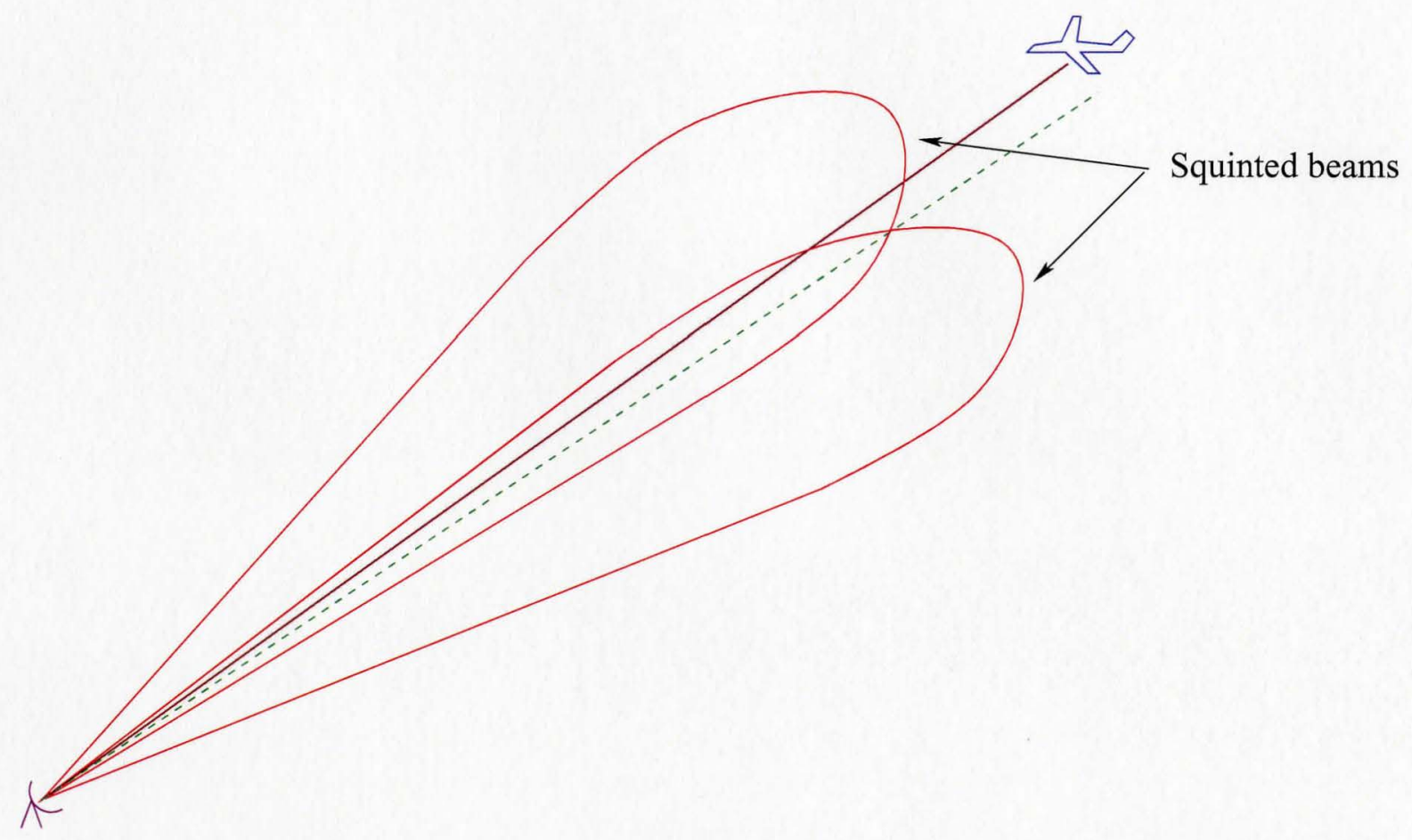

Figure 2.1: Monopulse radar beams

\subsection{Amplitude Comparison Monopulse Radar}

In an amplitude comparison monopulse radar system, a pulse energy is transmitted towards the targets and the target echoes are received with two beams (per angular dimension) that are squinted relative to the transmitted direction. Figure 2.1 shows the pair of beams required to measure a single angular coordinate. For the case of tracking targets in $3-D$ space, it requires four beams to measure bearing as well as elevation. In a conventional radar system, the direction of arrival (DOA) is estimated with the in-phase part (i.e., real part) of the monopulse ratio, which is formed by dividing the difference of the two received signals by their sum. 


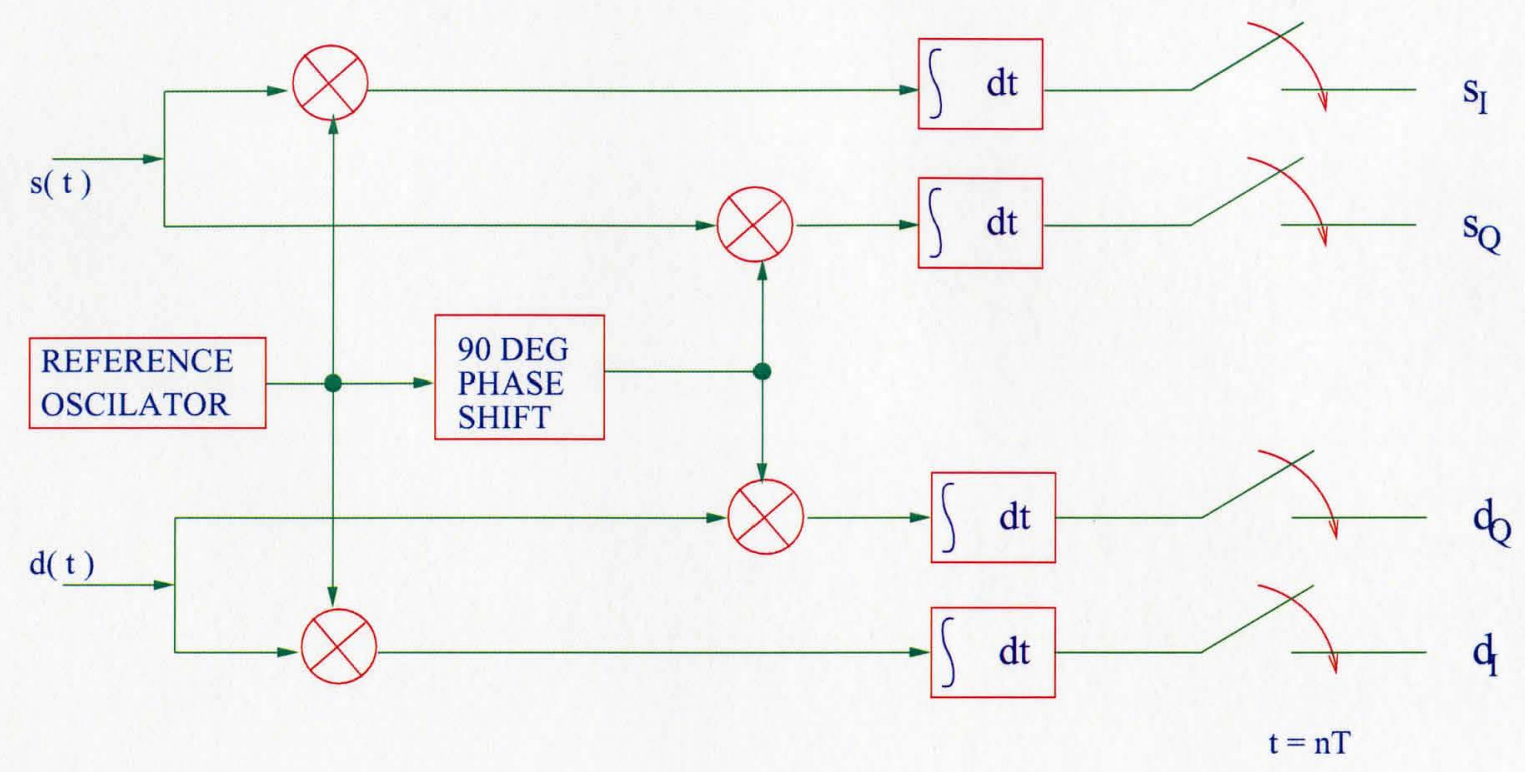

Figure 2.2: Monopulse radar signal processing for a single angular dimension

\subsection{Monopulse Radar Signal Processing}

The monopulse signal processor consists of a bank of matched filters that are fed with the sum and difference signals. This sum and difference signals are obtained using passive adders and subtractors at radio frequency to avoid drifts in active devices. Figure 2.2 shows a typical monopulse radar signal processor for a single angle measurement. The outputs comprises the in-phase sum $\left(s_{I}\right)$, Q-phase sum $\left(s_{Q}\right)$, in-phase difference $\left(d_{I}\right)$, and Q-phase difference $\left(d_{Q}\right)$. 


\subsection{Monopulse Signals with $N$ Unresolved Targets}

Consider the monopulse radar system with $N$ independent targets falling into a single range bin in $3-D$ space. The matched filter outputs comprises six signals and they can be written as

1. In-phase sum signal

$$
s_{I}=\sum_{i=1}^{N} \alpha_{i} \cos \phi_{i}+n_{s I}
$$

2. Quadrature sum signal

$$
s_{Q}=\sum_{i=1}^{N} \alpha_{i} \sin \phi_{i}+n_{s Q}
$$

3. In-phase bearing difference signal

$$
d_{b I}=\sum_{i=1}^{N} \eta_{b i} \alpha_{i} \cos \phi_{i}+n_{d b I}
$$

4. Quadrature bearing difference signal

$$
d_{b Q}=\sum_{i=1}^{N} \eta_{b i} \alpha_{i} \sin \phi_{i}+n_{d b Q}
$$

5. In-phase elevation difference signal

$$
d_{e I}=\sum_{i=1}^{N} \eta_{e i} \alpha_{i} \cos \phi_{i}+n_{d e I}
$$


6. Quadrature elevation difference signal

$$
d_{e Q}=\sum_{i=1}^{N} \eta_{e i} \alpha_{i} \sin \phi_{i}+n_{d e Q}
$$

where

$$
\begin{aligned}
\alpha_{i} & =\sqrt{\kappa} A_{i} G_{\Sigma}^{2}\left(\theta_{b i}, \theta_{e i}\right) p_{0} \quad \text { (signal amplitude of target } i \text { ) } \\
\eta_{b i} & \left.=\frac{G_{b \Delta}\left(\theta_{b i}, \theta_{e i}\right)}{G_{\Sigma}\left(\theta_{b i}, \theta_{e i}\right)} \quad \text { (bearing DOA of target } i\right) \\
\eta_{e i} & \left.=\frac{G_{e \Delta}\left(\theta_{b i}, \theta_{e i}\right)}{G_{\Sigma}\left(\theta_{b i}, \theta_{e i}\right)} \quad \text { (elevation DOA of target } i\right) \\
\kappa & =\text { constant proportional to transmitted power } \\
A_{i} & =\text { voltage amplitude of target } i \\
G_{\Sigma}\left(\theta_{b i}, \theta_{e i}\right) & =\text { sum channel voltage gain } \\
G_{b \Delta}\left(\theta_{b i}, \theta_{e i}\right) & =\text { bearing difference channel voltage gain } \\
G_{e \Delta}\left(\theta_{b i}, \theta_{e i}\right) & =\text { elevation difference channel voltage gain } \\
\theta_{b i} & =\text { bearing of the target } i \\
\theta_{e i} & =\text { elevation of the target } i \\
p_{0} & =\text { receiver matched filter gain } \\
\phi_{i} & =\text { phase angle of target } i \text { return } \\
n_{s I} & \sim \mathcal{N}\left(0, \sigma_{s}^{2}\right) \quad \text { sum in-phase noise } \\
n_{s Q} & \sim \mathcal{N}\left(0, \sigma_{s}^{2}\right) \quad \text { sum quadrature noise } \\
n_{d b I} & \sim \mathcal{N}\left(0, \sigma_{d}^{2}\right) \quad \text { bearing difference in phase noise } \\
n_{d b Q} & \sim \mathcal{N}\left(0, \sigma_{d}^{2}\right) \quad \text { bearing difference quadrature noise } \\
n_{d e I} & \sim \mathcal{N}\left(0, \sigma_{d}^{2}\right) \quad \text { elevation difference in phase noise }
\end{aligned}
$$




$$
n_{\text {deQ }} \sim \mathcal{N}\left(0, \sigma_{d}^{2}\right) \quad \text { elevation difference quadrature noise }
$$

with $\mathcal{N}\left(\mu, \sigma^{2}\right)$ denoting Gaussian distribution with mean $\mu$ and variance $\sigma^{2}$. The channel voltage gains $G_{\Sigma}\left(\theta_{b i}, \theta_{e i}\right), G_{b \Delta}\left(\theta_{b i}, \theta_{e i}\right)$ and $G_{e \Delta}\left(\theta_{b i}, \theta_{e i}\right)$ can be expressed in terms of individual beam voltage gain as follows

$$
\begin{aligned}
G_{\Sigma}\left(\theta_{b i}, \theta_{e i}\right)= & \Psi\left(s q_{e}, s q_{b}, \theta_{b i}, \theta_{e i}\right)+\Psi\left(-s q_{e}, s q_{b}, \theta_{b i}, \theta_{e i}\right) \\
& +\Psi\left(s q_{e},-s q_{b}, \theta_{b i}, \theta_{e i}\right)+\Psi\left(-s q_{e},-s q_{b}, \theta_{b i}, \theta_{e i}\right) \\
G_{b \Delta}\left(\theta_{b i}, \theta_{e i}\right)= & \Psi\left(s q_{e}, s q_{b}, \theta_{b i}, \theta_{e i}\right)+\Psi\left(-s q_{e}, s q_{b}, \theta_{b i}, \theta_{e i}\right) \\
& -\Psi\left(s q_{e},-s q_{b}, \theta_{b i}, \theta_{e i}\right)-\Psi\left(-s q_{e},-s q_{b}, \theta_{b i}, \theta_{e i}\right) \\
G_{e \Delta}\left(\theta_{b i}, \theta_{e i}\right)= & \Psi\left(s q_{e}, s q_{b}, \theta_{b i}, \theta_{e i}\right)-\Psi\left(-s q_{e}, s q_{b}, \theta_{b i}, \theta_{e i}\right) \\
& +\Psi\left(s q_{e},-s q_{b}, \theta_{b i}, \theta_{e i}\right)-\Psi\left(-s q_{e},-s q_{b}, \theta_{b i}, \theta_{e i}\right)
\end{aligned}
$$

where $s q_{e}$ and $s q_{b}$ are the squint angles for bearing and elevation, respectively, and are given by

$$
\begin{aligned}
& s q_{b}=s q_{0} / \cos \left(\hat{b}-b_{0}\right) \\
& s q_{e}=s q_{0} / \cos \left(\hat{e}-e_{0}\right)
\end{aligned}
$$

with $s q_{0}$ being the broadside squint angle, $b_{0}$ and $e_{0}$ are the bearing and elevation broadside angles, respectively, and $\hat{b}$ and $\hat{e}$ are the bearing and elevation beam-pointing angles, respectively. 
The beam voltage gain, $\Psi\left(x, y, \theta_{b i}, \theta_{e i}\right)$, can be written in terms of the normalized voltage patterns for bearing and elevation as follows:

$$
\Psi\left(x, y, \theta_{b i}, \theta_{e i}\right)=V_{b}\left(\theta_{b i}, \hat{b}+x\right) V_{e}\left(\theta_{e i}, \hat{e}+y\right)
$$

The normalized voltage patterns for the bearing and elevation, $V_{b}\left(\theta_{b i}, x\right)$ and $V_{e}\left(\theta_{e i}, y\right)$, are given by

$$
\begin{aligned}
& V_{b}\left(\theta_{b i}, x\right)=\frac{\pi}{4 V_{0}} \frac{\sin \left(N b_{2}\right)}{N \sin \left(b_{2}\right)}\left[\frac{\sin \left(b_{1}+0.5 \pi\right)}{b_{1}+0.5 \pi}+\frac{\sin \left(b_{1}-0.5 \pi\right)}{b_{1}-0.5 \pi}\right] \\
& V_{e}\left(\theta_{e i}, y\right)=\frac{\pi}{4 V_{0}} \frac{\sin \left(N e_{2}\right)}{N \sin \left(e_{2}\right)}\left[\frac{\sin \left(e_{1}+0.5 \pi\right)}{e_{1}+0.5 \pi}+\frac{\sin \left(e_{1}-0.5 \pi\right)}{e_{1}-0.5 \pi}\right]
\end{aligned}
$$

where

$$
\begin{aligned}
& V_{0}=\pi \frac{\sin \left(0.5 N \pi \sin \left(s q_{0}\right)\right)}{N \sin \left(0.5 \pi \sin \left(s q_{0}\right)\right)} \frac{\sin (0.5 \pi)}{0.5 \pi} \\
& b_{1}=0.25 \sin \left(\theta_{b i}-b_{0}\right) \\
& b_{2}=0.5\left[\sin \left(\theta_{b i}-b_{0}\right)-\sin \left(x-b_{0}\right)\right] \\
& e_{1}=0.25 \sin \left(\theta_{e i}-e_{0}\right) \\
& e_{2}=0.5\left[\sin \left(\theta_{e i}-e_{0}\right)-\sin \left(y-e_{0}\right)\right]
\end{aligned}
$$

with $V_{0}$ being the normalizing factor and $N$ is number of elements in the array along a single dimension.

Often, the $\mathrm{DOA}, \eta$, is approximated by a linear error function given by [30]

$$
\eta \approx k_{m} \theta, \quad-\theta_{B W}<\theta<\theta_{B W}
$$


where $\theta_{B W}$ is the two-way beamwidth, $k_{m}$ is the monopulse error slope, and $\theta$ is the AOA. The values of the monopulse error slope employed in the radar model used in this thesis for bearing and elevation, $k_{m}^{b}$ and $k_{m}^{e}$, respectively, are given by

$$
\begin{aligned}
& k_{m}^{b}=k_{0} \cos \left(\hat{b}-b_{0}\right) \operatorname{rad}^{-1} \\
& k_{m}^{e}=k_{0} \cos \left(\hat{e}-e_{0}\right) \operatorname{rad}^{-1}
\end{aligned}
$$

where $k_{0}=53.9$.

\subsection{Distribution of Monopulse Signals}

In this thesis, we consider Swerling I targets whose RCS fluctuation is described by Rayleigh distribution. The signal amplitude, $\alpha_{i}$ in (2.7), that is proportional to target RCS, has Rayleigh distribution too. The phase angle of the target return, $\phi_{i}$ in (2.18), is usually assumed to be uniformly distributed in $[0,2 \pi]$. Therefore, it can be easily shown that each summand in (2.1) - (2.6) is a Gaussian random variable [16]. Hence $z=\left[\begin{array}{llllll}s_{I} & d_{b I} & d_{e I} & s_{Q} & d_{b Q} & d_{e Q}\end{array}\right]^{T}$

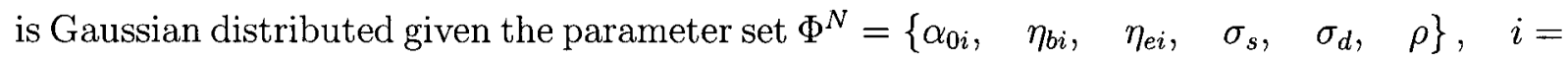
$1,2, \ldots, N$. It can be written as

$$
p\left(z \mid \Phi^{N}\right)=\mathcal{N}(z ; 0, P)
$$

where the covariance matrix $P$ is given by

$$
P=\left[\begin{array}{cc}
P_{1} & 0 \\
0 & P_{1}
\end{array}\right]
$$


The off-diagonal blocks are zero matrices since there is no correlation between the in-phase and quadrature-phase signals of the matched filter. The diagonal blocks are identical and given by

$$
P_{1}=\left[\begin{array}{ccc}
a_{11} & a_{12} & a_{13} \\
a_{12} & a_{22} & a_{23} \\
a_{13} & a_{23} & a_{33}
\end{array}\right]
$$

where the matrix elements are given by

$$
\begin{aligned}
& a_{11}=\sum_{i=1}^{N} \alpha_{0 i}^{2}+\sigma_{s}^{2} \\
& a_{12}=\sum_{i=1}^{N} \alpha_{0 i}^{2} \eta_{b i}+\rho \sigma_{s} \sigma_{d} \\
& a_{13}=\sum_{i=1}^{N} \alpha_{0 i}^{2} \eta_{e i}+\rho \sigma_{s} \sigma_{d} \\
& a_{22}=\sum_{i=1}^{N} \alpha_{0 i}^{2} \eta_{b i}^{2}+\sigma_{d}^{2} \\
& a_{23}=\sum_{i=1}^{N} \alpha_{0 i}^{2} \eta_{b i} \eta_{e i}+\rho \sigma_{d}^{2} \\
& a_{33}=\sum_{i=1}^{N} \alpha_{0 i}^{2} \eta_{e i}^{2}+\sigma_{d}^{2}
\end{aligned}
$$

In the above, $\alpha_{0 i}$ is the average value of target amplitude $\alpha_{i}$. The correlation coefficient $\rho$ is included to allow for a real-valued correlation between the receiver errors that may result from the local oscillator. 


\section{Chapter 3}

\section{Detection and Tracking}

Since this thesis is concerned with unresolved target detection and tracking, this Chapter is dedicated to review the basic theory behind the detection and tracking. Figure 3.3 shows the block diagram of a tracking system. There are two primary blocks, which are concerned with this thesis, namely the signal processor and the information processor. The signal processor involves complex signal processing of noisy electromagnetic or acoustic signals and followed by detection. The information processor involves data association and followed by track filtering. The main issues related to this thesis namely detection theory, filtering and data association are briefly reviewed in section $3.1,3.2$ and 3.3 respectively.

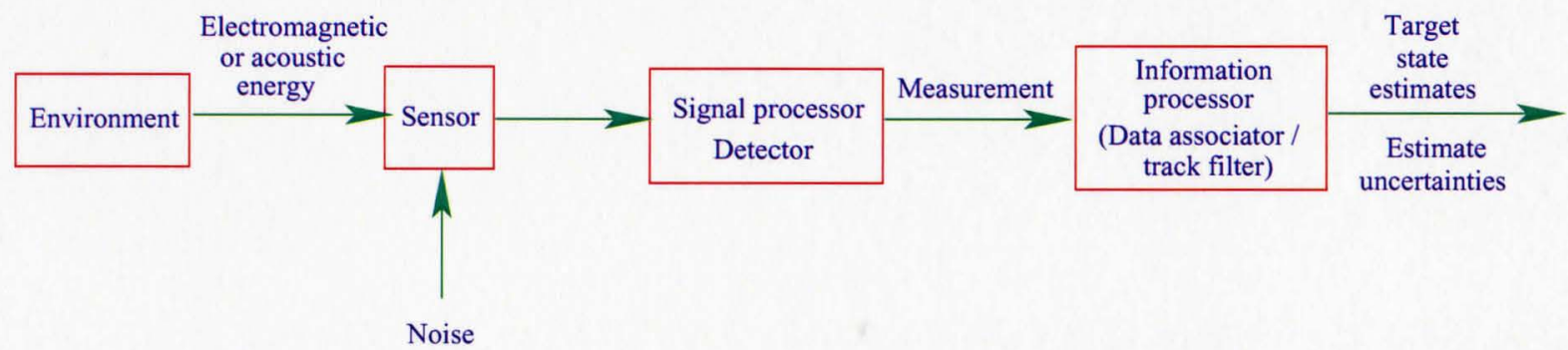

Figure 3.3: A typical tracking system 


\subsection{Detection Theory}

This section reviews the fundamentals of detection theory. Here, a brief overview is presented while the complete theory behind detection can be found in [28]. In a statistical decision problem, a decision has to be made as to which of several hypotheses is the correct one. A hypothesis is one of the possible events that can arise in the statistical decision problem. In general, there may be $M$ hypotheses from which correct one has to be selected. Next two subsections brief on the simplest case where there are two possible hypotheses and general case where more than two hypotheses are possible respectively.

\subsubsection{Binary Hypothesis Test}

In a binary testing problem, we need to decide on choice $D_{i}$ based on observation $z$ while the true hypothesis is denoted by $H_{j}$, where $i, j=0,1$. There are four possibilities, (i) $H_{0}$ is true, decide $D_{0}$ (ii) $H_{1}$ is true, decide $D_{1}$ (iii) $H_{0}$ is true, decide $D_{1}$ (iv) $H_{1}$ is true, decide $D_{0}$. The first two are correct choices whereas the last two are errors. With the a priori probabilities $P\left(H_{0}\right)$ and $P\left(H_{1}\right)$, and the associated cost $C_{i j}$ for making a decision $D_{i}$ when the true hypothesis is $H_{j}$, the average cost, $\bar{C}$, can be written as,

$$
\bar{C}=\sum_{i=0}^{1} \sum_{j=0}^{1} C_{i j} P\left(D_{i} \mid H_{j}\right) P\left(H_{j}\right)
$$

where $P\left(D_{i} \mid H_{j}\right)$ is the probability of deciding $D_{i}$ while $H_{j}$ is true and can be obtained by integrating the density function of $p\left(z \mid H_{j}\right)$ over $Z_{i}$, the decision region corresponding to $D_{i}$, i.e.

$$
P\left(D_{i} \mid H_{j}\right)=\int_{Z_{i}} p\left(z \mid H_{j}\right) d z
$$


Thus the average cost becomes

$$
\bar{C}=\sum_{i=0}^{1} \sum_{j=0}^{1} C_{i j} P\left(H_{j}\right) \int_{Z_{i}} p\left(z \mid H_{j}\right) d z
$$

Noting that the decision spaces $Z_{0}$ and $Z_{1}$ are exclusive and exhaustive, it can be further simplified as,

$$
\begin{aligned}
\bar{C}= & C_{10} P\left(H_{0}\right)+C_{11} P\left(H_{1}\right) \\
& +\int_{Z_{0}}\left[P\left(H_{1}\right)\left(C_{01}-C_{11}\right) p\left(z \mid H_{1}\right)-P\left(H_{0}\right)\left(C_{10}-C_{00}\right) p\left(z \mid H_{0}\right)\right] d z
\end{aligned}
$$

For the Bayes criterion, the average cost should be minimized. Noting that first two terms are fixed, minimizing $\bar{C}$ is equivalent to select the region, $Z_{1}$, such that

$$
P\left(H_{1}\right)\left(C_{01}-C_{11}\right) p\left(z \mid H_{1}\right) \geq P\left(H_{0}\right)\left(C_{10}-C_{00}\right) p\left(z \mid H_{0}\right)
$$

Hence the likelihood ratio test yields the decision

$$
\Lambda(z) \triangleq \frac{p\left(z \mid H_{1}\right)}{p\left(z \mid H_{0}\right)} \underset{H_{0}}{\gtrless} \frac{P\left(H_{0}\right)\left(C_{10}-C_{00}\right)}{P\left(H_{1}\right)\left(C_{01}-C_{11}\right)} \triangleq \eta
$$

Similarly, other criterions (Maximum A Posterior, Minimax, Neyman-Pearson) which are special cases of the Bayes criterions end up with likelihood ratio tests. 


\subsubsection{Multiple Hypotheses Test}

In many situations, there are several alternatives from which one of the hypotheses have to be selected. Similar to binary case, for $M$ hypotheses testing, one can write the average cost with given the a priori probabilities, $P\left(H_{0}\right), P\left(H_{1}\right), \ldots, P\left(H_{M-1}\right)$, and associated cost $C_{i j}$ for choosing $H_{i}$ when the correct hypothesis is $H_{j}$, i.e.,

$$
\vec{C}=\sum_{i=0}^{M-1} \sum_{j=0}^{M-1} C_{i j} P\left(H_{i} \mid H_{j}\right) P\left(H_{j}\right)
$$

With the similar arguments as in binary case, it can be written as,

$$
\bar{C}=\sum_{i=0}^{M-1} C_{i i} P\left(H_{i}\right)+\sum_{i=0}^{M-1} \int_{Z_{i}} \sum_{j=0, j \neq i}^{M-1} P\left(H_{j}\right)\left(C_{i j}-C_{j j}\right) p\left(z \mid H_{j}\right) d z
$$

The first term represents the fixed cost and let's define the term inside the integral of the second term as

$$
I_{i}(z)=\sum_{j=0, j \neq i}^{M-1} P\left(H_{j}\right)\left(C_{i j}-C_{j j}\right) p\left(z \mid H_{j}\right)
$$

By observing (3.44), it is obvious that we have to decide on the hypothesis which corresponds to the minimum value of $I_{i}(z)$ as the correct one.

An important special case, which is used in this thesis, is when

$$
P\left(H_{0}\right)=P\left(H_{1}\right)=\cdots=P\left(H_{M-1}\right)=P, \quad C_{i j}=1 \text { for } i \neq j \text { and } C_{i i}=0
$$


in which case we have

$$
I_{i}(z)=\sum_{j=0, j \neq i}^{M-1} p\left(z \mid H_{j}\right) P=\left[1-p\left(z \mid H_{i}\right)\right] P
$$

Then the decision rule is to choose $H_{i}$ for which $p\left(z \mid H_{i}\right)$ is maximum.

\section{$3.2 \quad$ Filtering}

Once the measurements are associated with tracks, which is to be discussed in section 3.3, track filters are used to estimate the target states. The recursive Bayesian type filters are easy to implement and well established. Here some well known recursive filters are reviewed. They work well in different settings and assumptions. The Kalman filter which is optimum in linear, Gaussian problems is given in section 3.2.1. In section 3.2.2, another optimal filter, grid based filter, is reviewed. Extended Kalman filter (EKF), a variant of the Kalman filter, is given in section 3.2.3. Finally particle filter which has proven to be effective for nonlinear non-Gaussian problems is given in section 3.2.4.

\subsubsection{Kalman Filter}

If some assumptions hold, the Kalman Filter completely and exactly characterizes the probability distribution of the state. It is then the optimal method for conducting sequential Bayesian inference. The assumptions of Kalman filter are:

- $p\left(\mathbf{x}_{0}\right)=\mathcal{N}\left(\mathbf{m}_{0}, C_{0}\right)$

- $p\left(\mathbf{x}_{k} \mid \mathbf{x}_{k-1}\right)=\mathcal{N}\left(f_{k}\left(\mathbf{x}_{k-1}\right), \Sigma_{k}\right)$

- $p\left(\mathbf{z}_{k} \mid \mathbf{x}_{k}\right)=\mathcal{N}\left(h_{k}\left(\mathbf{x}_{k}\right), \Gamma_{k}\right)$ 
- $f_{k}\left(\mathbf{x}_{k}\right)$ and $h_{k}\left(\mathbf{x}_{k}\right)$ are known linear functions of $\mathbf{x}_{k}$.

- $m_{0}, C_{0}, \Sigma_{k}$ and $\Gamma_{k}$ are known.

- $\mathbf{x}_{k}-f_{k}\left(\mathbf{x}_{k-1}\right)$ and $\mathbf{z}_{k}-h_{k}\left(\mathbf{x}_{k}\right)$ are uncorrelated.

where $\mathcal{N}(\mathrm{m}, P)$ is a Gaussian distribution characterized by its mean, $\mathrm{m}$, and covariance, $P$. It is worth noting also that the final assumption can be circumvented by transforming the variables such that the assumption holds.

The Kalman filter works because, if $p\left(\mathbf{x}_{k-1} \mid \mathbf{z}_{1: k-1}\right)$ and $p\left(\mathbf{x}_{k} \mid \mathbf{x}_{k-1}\right)$ and $p\left(\mathbf{z}_{k} \mid \mathbf{x}_{k}\right)$ are all Gaussian distributions then so is $p\left(\mathbf{x}_{k} \mid \mathbf{z}_{1: k}\right)$. It is possible to prove the same recursion using a Least Squares (LS) argument, through such an approach recursively describes, not characterizes, the distribution.

$$
\begin{aligned}
p\left(\mathbf{x}_{k-1} \mid \mathbf{z}_{1: k-1}\right) & =\mathcal{N}\left(\mathbf{m}_{k-1 \mid k-1}, C_{k-1 \mid k-1}\right) \\
p\left(\mathbf{x}_{k} \mid \mathbf{z}_{1: k-1}\right) & =\mathcal{N}\left(\mathbf{m}_{k \mid k-1}, C_{k \mid k-1}\right) \\
& =\mathcal{N}\left(f_{k}\left(\mathbf{m}_{k-1 \mid k-1}\right), \Sigma_{k}+F_{k} C_{k-1 \mid k-1} F_{k}^{T}\right) \\
p\left(\mathbf{x}_{k} \mid \mathbf{z}_{1: k}\right) & =\mathcal{N}\left(\mathbf{m}_{k \mid k}, C_{k \mid k}\right) \\
& =\mathcal{N}\left(\mathbf{m}_{k \mid k-1}+W_{k} \tilde{\mathbf{z}}_{k \mid k-1}, C_{k \mid k-1}-W_{k} H_{k} C_{k \mid k-1}\right)
\end{aligned}
$$

where $\mathbf{m}_{k \mid k^{\prime}}$ and $C_{k \mid k^{\prime}}$ are respectively the mean and covariance of the distribution of the state at time $k$ given data up to time $k^{\prime}$. The functions $f_{k}\left(X_{k}\right)$ and $h_{k}\left(X_{k}\right)$ are linear functions, which can include bias terms. $\Omega_{k}$ and $W_{k}$ are the covariance of the innovation term, $\tilde{\mathbf{z}}_{k \mid k-1}$, and the Kalman gain, respectively. 


$$
\begin{aligned}
\tilde{\mathbf{z}}_{k \mid k-1} & =\mathbf{z}_{k}-\hat{\mathbf{z}}_{k \mid k-1} \\
\hat{\mathbf{z}}_{k \mid k-1} & =h_{k}\left(\mathbf{m}_{k \mid k-1}\right) \\
F_{k} & =\left.\frac{d f_{k}\left(\mathbf{x}_{k-1}^{\prime}\right)}{d \mathbf{x}_{k}^{\prime}}\right|_{\mathbf{x}_{k-1}^{\prime}=\mathbf{m}_{k-1 \mid k-1}} \\
H_{k} & =\left.\frac{d h_{k}\left(\mathbf{x}_{k}^{\prime}\right)}{d \mathbf{x}_{k}^{\prime}}\right|_{\mathbf{x}_{k}^{\prime}=\mathbf{m}_{k \mid k-1}} \\
\Omega_{k} & =H_{k} C_{k \mid k-1} H_{k}^{T}+\Gamma_{k} \\
W_{k} & =C_{k \mid k-1} H_{k}^{T} \Omega_{k}^{-1}
\end{aligned}
$$

An interesting point to note about the recursion formed by (3.47) and (3.48) is that there is no effect of the measurement on the covariances of the normal distributions. The covariance is defined entirely by the matrices the comprise the system, $F_{k}, H_{k}, \Sigma_{k}, \Gamma_{k}$, $C_{0}$. If the system matrices are known and constant over time, then the covariances can be precalculated. It is also worth noting that in such cases, the covariance will tend to a value. This means that, after some initial period, the uncertainty is constant. This steady state is the result of a balance between the increase in uncertainty as a result of the prediction step and the reduction in uncertainty due to the update step. This observation leads to the $\alpha-\beta$ filter, which can be viewed as a special case of the Kalman filter.

\subsubsection{Grid Based Filter}

The Kalman filter is not the only optimal algorithm for sequential inference. The grid based filter is another example of an optimal recursion resulting from a different set of assumptions.

- The state space is discrete and at any time, $k-1$, consists of a finite number, $N_{P}$, of 
state at $\mathbf{x}_{k-1}^{j}, j=1: N_{P}$.

- $p\left(\mathbf{x}_{0}\right)=\sum_{j=1}^{N_{P}} w_{0 \mid 0}^{j} \delta\left(\mathbf{x}_{0}-\mathbf{x}_{0}^{j}\right)$

- $p\left(\mathbf{x}_{k}^{j} \mid \mathbf{x}_{k-1}^{l}\right)$ and $p\left(\mathbf{z}_{k} \mid \mathbf{x}_{k}^{j}\right)$ are known.

For each state $\mathbf{x}_{k-1}^{j}$, let the conditional probability of that state, given measurements up to time $k-1$ be denoted by $w_{k-1 \mid k-1}^{j}$. Then, a recursion can be derived for the posterior pdf at time $k-1$.

$$
\begin{aligned}
p\left(\mathbf{x}_{k-1} \mid \mathbf{z}_{1: k-1}\right) & =\sum_{j=1}^{N_{P}} w_{k-1 \mid k-1}^{j} \delta\left(\mathbf{x}_{k-1}-\mathbf{x}_{k-1}^{j}\right) \\
p\left(\mathbf{x}_{k} \mid \mathbf{z}_{1: k-1}\right) & =\sum_{j=1}^{N_{P}} w_{k \mid k-1}^{j} \delta\left(\mathbf{x}_{k}-\mathbf{x}_{k}^{j}\right) \\
p\left(\mathbf{x}_{k} \mid \mathbf{z}_{1: k}\right) & =\sum_{j=1}^{N_{P}} w_{k \mid k}^{j} \delta\left(\mathbf{x}_{k}-\mathbf{x}_{k}^{j}\right)
\end{aligned}
$$

The weights can be calculated in terms of the transition and observation probabilities.

$$
\begin{aligned}
w_{k \mid k-1}^{j} & =\sum_{l=1}^{N_{P}} w_{k-1 \mid k-1}^{l} p\left(\mathbf{x}_{k}^{j} \mid \mathbf{x}_{k-1}^{l}\right) \\
w_{k \mid k}^{j} & =\frac{w_{k \mid k-1}^{j} p\left(\mathbf{z}_{k} \mid \mathbf{x}_{k}^{j}\right)}{\sum_{l=1}^{N_{P}} w_{k \mid k-1}^{l} p\left(\mathbf{z}_{k} \mid \mathbf{x}_{k}^{l}\right)}
\end{aligned}
$$

The above does assume that $p\left(\mathbf{x}_{k}^{j} \mid \mathbf{x}_{k-1}^{l}\right)$ and $p\left(\mathbf{z}_{k} \mid \mathbf{x}_{k}^{j}\right)$ are known, but does not constrain the particular form of these discrete distributions. 


\subsubsection{Extended Kalman Filter}

In many situations of interest, neither of these sets of assumptions hold. It is then necessary to make approximation. If $p\left(\mathbf{x}_{0}\right), p\left(\mathbf{x}_{k} \mid \mathbf{x}_{k-1}\right)$ and $p\left(\mathbf{z}_{k} \mid \mathbf{x}_{k}\right)$ are approximated as Gaussian and $f_{k}\left(\mathbf{x}_{k-1}\right)$ and $h_{k}\left(\mathbf{x}_{k}\right)$ are approximated as linear, then the recursion described above becomes the Extended Kalman Filter, EKF.

$$
\begin{aligned}
p\left(\mathbf{x}_{k-1} \mid \mathbf{z}_{1: k-1}\right) & \approx \mathcal{N}\left(\mathbf{m}_{k-1 \mid k-1}, C_{k-1 \mid k-1}\right) \\
p\left(\mathbf{x}_{k} \mid \mathbf{z}_{1: k-1}\right) & \approx \mathcal{N}\left(\mathbf{m}_{k \mid k-1}, C_{k \mid k-1}\right) \\
& \approx \mathcal{N}\left(f_{k}\left(\mathbf{m}_{k-1 \mid k-1}\right), \Sigma_{k}+F_{k} C_{k-1 \mid k-1} F_{k}^{T}\right) \\
p\left(\mathbf{x}_{k} \mid \mathbf{z}_{1: k}\right) & \approx \mathcal{N}\left(\mathbf{m}_{k \mid k}, C_{k \mid k}\right) \\
& \approx \mathcal{N}\left(\mathbf{m}_{k \mid k-1}+W_{k} \tilde{\mathbf{z}}_{k \mid k-1}, C_{k \mid k-1}-W_{k} H_{k} C_{k \mid k-1}\right)
\end{aligned}
$$

Such a local approximation of the equations may be a sufficient description of the nonlinearity. However, it is common that it is not. A better approximation can be made by considering the above approximation as using the first term in Taylor expansions of the nonlinear functions, $f_{k}\left(\mathbf{x}_{k-1}\right)$ and $h_{k}\left(\mathbf{x}_{k}\right)$. A higher order EKF that retains further terms in the Taylor expansions exists and results in a closer approximation to the true posterior. The additional complexity has prohibited its widespread use.

Recently, the unscented transform has been used in an EKF framework [11]. This approach considers a set of points that are deterministically sampled from Gaussian approximations to $p\left(\mathbf{x}_{k-1} \mid \mathbf{z}_{1: k-1}\right)$. These points are all propagated through the true nonlinearity and the parameters of the Gaussian approximation are re-estimated. This filter is equivalent to a higher order EKF and its algorithmic simplicity makes it more attractive that the higher order EKF.

If the true distribution of non-Gaussian then a Gaussian, however good approximation, 
can never describe the distribution well. It is possible to approximate the state space as consisting of a grid of points and then use an approximate grid based approach. This is frequently the approach taken by the speech processing research community. In such cases, approximate grid based filters yield an improvement in performance in comparison to that of EKF [1].

\subsubsection{Particle Filter}

In traditional state estimation problem, the Kalman Filter (KF) which is the best algorithm for linear Gaussian system, is used extensively. It fails for nonlinear system and its variant, Extended Kalman Filter (EKF) which approximates the system models to fit into linear Gaussian frame work, also fails for the case of high nonlinear and non-Gaussian system. Rather than approximating the models in order to be able to fit a distribution of a given type to the posterior, a particle filter explicitly approximates the distribution so that it can handle high nonlinear non-Gaussian models. The approach has also been known as bootstrap filter [9], condensation algorithm [14] and sequential Monte Carlo filtering [8].

In particle filtering, the required posterior density function is represented by a set of random samples ('particles') with associated weights [2] [9]. Let $\left\{\mathbf{x}_{k-1}^{j}\right\}_{j=1}^{N_{P}}$, with associated weights $\left\{w_{k-1}^{j}\right\}_{j=1}^{N_{P}}$ be the random samples representing the posterior density $p\left(\mathbf{x}_{k-1} \mid \mathbf{z}_{1: k-1}\right)$ of the state vector $\mathbf{x}_{k}$ at time epoch $k$, where $\mathbf{z}_{1: k-1}$ is the set of all measurements available at time $k$. The weights are normalized such that $\sum_{j=1}^{N_{P}} w_{k-1}^{j}=1$. There are many variants in particle filter. The simplest and widely implemented variant is Sampling Importance Resampling (SIR) particle filter which is also used in this thesis. 


\section{SIR Particle Filter}

Sampling Importance Resampling (SIR) [21], [26] propagates and updates the particles in which the $p\left(\mathbf{x}_{k-1} \mid \mathbf{z}_{1: k-1}\right)$ is represented by equally weighted particles. Then,

$$
p\left(\mathbf{x}_{k-1} \mid \mathbf{z}_{1: k-1}\right) \approx \frac{1}{N_{P}} \sum_{j=1}^{N_{P}} \delta\left(\mathbf{x}_{k-1}-\mathbf{x}_{k-1}^{j}\right)
$$

where $\delta(\cdot)$ is the Dirac Delta function. In this thesis, the prior density, $p\left(\mathbf{x}_{k-1} \mid \mathbf{z}_{1: k}\right)$ is chosen as the Importance Density. The propagation and update of the particles in SIR method are given as follows.

Prediction Take each existing sample, $\mathbf{x}_{k}^{j}$ and augment it with a sample $\mathbf{x}_{k}^{* j} \sim p\left(\mathbf{x}_{k} \mid \mathbf{x}_{k-1}^{j}\right)$, using the system model. The set $\left\{\mathbf{x}_{k}^{* j}\right\}_{j=1}^{N_{P}}$ gives us an approximation of the prior, $p\left(\mathbf{x}_{k} \mid \mathbf{z}_{1: k-1}\right)$, at time $k$, i.e.

$$
p\left(\mathbf{x}_{k} \mid \mathbf{z}_{1: k-1}\right) \approx \frac{1}{N_{P}} \sum_{j=1}^{N_{P}} \delta\left(\mathbf{x}_{k}-\mathbf{x}_{k}^{* j}\right)
$$

Update Importance Weights: At each measurement epoch, to account for the fact that the samples, $\mathbf{x}_{k+1}^{* j}$ are not drawn from $p\left(\mathbf{x}_{k} \mid \mathbf{z}_{1: k}\right)$, the weights are modified using the principle of Importance Sampling. When using the prior as the Importance Density, it can be shown that the weights are given by

$$
w_{k}^{j} \propto p\left(\mathbf{z}(k) \mid \mathbf{x}_{k}=\mathbf{x}_{k}^{* j}, \mathbf{z}_{1: k-1}\right)
$$


Reselection: Resample (with replacement) from $\left\{\mathrm{x}_{k}^{* j}\right\}_{j=1}^{N_{P}}$, using the weights, $\left\{w_{k}^{* j}\right\}_{j=1}^{N_{P}}$, to generate a new sample, $\left\{\mathbf{x}_{k}^{j}\right\}_{j=1}^{N_{P}}$, then set $w_{k}^{j}=1 / N_{P}$ for $j=1, \ldots, m$. We then have:

$$
p\left(\mathbf{x}_{k} \mid \mathbf{z}_{1: k}\right) \approx \frac{1}{N_{P}} \sum_{j=1}^{N_{P}} \delta\left(\mathbf{x}_{k}-\mathbf{x}_{k}^{j}\right)
$$

At each stage the mean of the posterior distribution is used to estimate, $\hat{\mathbf{x}}_{k-1}$ of the target state, $\mathbf{x}_{k-1}$, i.e.

$$
\begin{aligned}
\hat{\mathbf{x}}_{k} & =\mathbb{E}\left[\mathbf{x}_{k} \mid \mathbf{z}_{1: k}\right] \\
& =\int_{\mathbf{x}_{k}} \mathbf{x}_{k} p\left(\mathbf{x}_{k} \mid \mathbf{z}_{1: k}\right) d \mathbf{x}_{k} \\
& \approx \frac{1}{N_{P}} \sum_{j=1}^{N_{P}} \mathbf{x}_{k}^{j}
\end{aligned}
$$

We use regularization to over come the degeneracy problem of the SIR filter.

\subsection{Data Association}

The data association problem is that of associating the many measurements made by a sensor with the underlying states or tracks that being observed. It includes issues of validating the correct measurement to the correct states or tracks, and initializing, confirming or deleting tracks or states. Whereas conventional estimation (discussed in section 3.2) is concerned with uncertainty in measurement localization, data association is concerned with uncertainty in measurement origin. Section 3.3.1 introduces a common measurement validation technique. The goal is to reduce computational costs by avoiding having to search for the measurements in the entire measurement space. The Probabilistic Data Association Filter (PDAF) for single targets and the Joint Probabilistic Data Association Filter (JPDAF) for multiple 
targets are reviewed in section 3.3 .2 and 3.3 .3 respectively.

\subsubsection{Gating}

In tracking applications, following the signal detection process that yields measurements, there is a procedure that selects the measurements to be incorporated into the state estimator. A gate is set up and the detection within this gate can be associated with the target of interest. If there is more than one measurement in the gate, this leads to an association uncertainty. Usually the gate is formed in terms of the normalized innovation $\nu_{k}$ which is defined as,

$$
\nu_{k}^{2}=\tilde{\mathbf{z}}_{k \mid k-1}^{T} \Omega_{k}^{-1} \tilde{\mathbf{z}}_{k \mid k-1}
$$

where $\tilde{\mathbf{z}}_{k \mid k-1}$ and $\Omega_{k}$ are in the innovation of the given measurement and innovation covariance and defined in (3.49) and (3.53) respectively. Assume that the true measurement, $\mathbf{Z}_{k}$, is Gaussian distributed with its pdf given by:

$$
p\left(\mathbf{z}_{k} \mid \mathbf{z}_{1: k-1}\right)=\mathcal{N}\left(\hat{\mathbf{z}}_{k \mid k-1}, \Omega_{k}\right)
$$

The pdf of the innovation, $\tilde{\mathbf{z}}_{k \mid k-1}$, is

$$
p\left(\tilde{\mathbf{z}}_{k \mid k-1}\right)=\frac{1}{(2 \pi)^{d / 2}\left|\Omega_{k}\right|^{1 / 2}} \exp \frac{-\nu_{k}^{2}}{2}
$$

where $d$ is the dimension of the measurement vector and $\left|\Omega_{k}\right|$ is the determinant of $\Omega_{k}$. Thus, the true measurement will be in the following region:

$$
\mathcal{G}(\gamma)=\left\{\mathbf{z}: \nu^{2} \leq \gamma\right\}
$$


with probability determined by the gate threshold $\gamma$. The region is called gate. The simplest method for data association is the Nearest Neighbor data association. It considers the measurement which is shortest distance from predicted mean as the associated measurement.

\subsubsection{Probabilistic Data Association (PDA)}

In case of a single target in clutter, more than one measurements may be validated that fall into the gate. Rather than taking a single measurement, it updates the track with all the validated measurement. It calculates the association probabilities for each validated measurement at the current step to the target of interest. This probabilistic (Bayesian) information leads to a tracking filter called PDA filter (PDAF), that accounts for the measurement origin uncertainty.

\section{Assumptions:}

- There is only one target of interest

- the true measurement is Gaussian distributed with pdf

$$
p\left(\mathbf{z}_{k} \mid \mathbf{z}_{1: k-1}\right)=\mathcal{N}\left(\hat{\mathbf{z}}_{k \mid k-1}, \Omega_{k}\right)
$$

- At each time step a gating region is set up

- At most, one measurement can be target originated. The remaining measurements are assumed due to false alarm or clutter.

- The target detections occur independently over time with known probability $P_{D}$ 
The latest set of validated measurements at time step $k$ is denoted as

$$
\mathbf{Z}_{k} \triangleq\left\{\mathbf{z}_{k, 1}, \cdots, \mathbf{z}_{k, m_{k}}\right\}=\left\{\mathbf{z}_{k, j}\right\}_{j=1}^{m_{k}}
$$

where each measurement $\mathbf{z}_{k, j}$ is the $j$ - th validated measurement. $m_{k}$ is the number of measurements in the validation region at time step $k$. The sequence of all measurements is denoted as

$$
\mathbf{Z}_{1: k} \triangleq\left\{\mathbf{Z}_{1}, \cdots, \mathbf{Z}_{k}\right\}=\left\{\mathbf{Z}_{j}\right\}_{j=1}^{k}
$$

To model the situation in which a target has not been detected, we introduce $z_{k, 0}$ as a Null-measurement. Furthermore, we define the association events $\theta_{k, j}$ as

$$
\theta_{k, j}=\left\{\mathbf{z}_{k, j} \text { is the target associated measurement }\right\} \quad j=0, \ldots, m_{k}
$$

The association probability $\beta_{k, j}$ is defined as the conditional probability of an association event

$$
\beta_{k, j} \triangleq P\left(\theta_{k, j} \mid \mathbf{z}_{k, j}\right)
$$

and by using the total probability theorem, the conditional mean of the state at time step $k$ can be written as

$$
\begin{aligned}
\hat{\mathbf{x}}_{k \mid k} & =E\left[\mathbf{x}_{k} \mid \mathbf{Z}_{1: k}\right] \\
& =\sum_{j=1}^{m_{k}} E\left[\mathbf{x}_{k} \mid \theta_{k, j}, \mathbf{Z}_{1: k}\right] \beta_{k, j} \\
& =\sum_{j=1}^{m_{k}} \hat{\mathbf{x}}_{k \mid k, j} \beta_{k, j}
\end{aligned}
$$

where $\hat{\mathbf{x}}_{k \mid k, j}$ is the a posteriori state conditioned on the event $\theta_{k, j}$. 


\subsubsection{Joint Probabilistic Data Association (JPDA)}

The Joint Probabilistic Data Association (JPDA) approach is the extension of the PDA approach. The same assumptions as in the PDA approach are to be considered, besides that there is a known number of targets $N_{T}$ instead of a single target. The index $t$ designates one among the $N_{T}$ targets. In the JPDA approach, the measurement to target association probabilities are evaluated jointly across the targets. After gating, a validation matrix that indicates all the possible sources of each measurement can be set up. From this validation matrix all the feasible joint association events are obtained according to the rules

- one source for each measurement

- one measurement (or none) from each target

Let $\theta$ denote the joint association event (the time index is omitted for simplicity where it does not confuse) and $\theta_{j, t}$ is the particular event which assigns measurement $j$ to target $t$. To evaluate the marginal association probabilities

$$
\beta_{j, t} \triangleq P\left(\theta_{j, t} \mid \mathbf{Z}_{1: k}\right)
$$

it is assumed that the estimation problem is Markovian, thus the joint association probabilities are given by

$$
\begin{aligned}
P\left(\theta \mid \mathbf{Z}_{1: k}\right) & =P\left(\theta \mid \mathbf{Z}_{k}, \mathbf{Z}_{1: k-1}\right) \\
& =P\left(\theta \mid \mathbf{Z}_{k}, \mathbf{x}_{1: N_{T}}\right) \\
& =\frac{1}{c} p\left(\mathbf{Z}_{k} \mid \theta, \mathbf{x}_{1: N_{T}}\right) P\left(\theta \mid \mathbf{x}_{1: N_{T}}\right)
\end{aligned}
$$


where $c$ is a normalization constant. The term $P\left(\theta \mid \mathbf{x}_{1: N_{T}}\right)$ corresponds to the probability of the assignment $\theta$ given the current states of the targets. We approximate this term by

$$
P\left(\theta \mid \mathbf{x}_{1: N_{T}}\right)=P_{D}^{N_{T}-n}\left(1-P_{D}\right)^{n}
$$

where $P_{D}$ is the probability of detection, and $n$ is the number of assigned Null-measurements $\mathbf{z}_{0}$. By assuming that the measurements are detected independently of each other, we get

$$
p\left(\mathbf{Z}_{k} \mid \theta, \mathbf{x}_{1: N_{T}}\right)=P_{F A}^{m_{k}-\left(N_{T}-n\right)} \prod_{\theta_{j, t} \in \theta} p_{j, t}
$$

where $P_{F A}$ is the probability of false alarm and $p_{j, t}$ is the association likelihood that measurement $j$ assigns target $t$. It is determined by

$$
p_{j, t}=\frac{1}{(2 \pi)^{d / 2}\left|\Omega_{j, t}\right|^{1 / 2}} \exp \frac{-\nu_{j, t}^{2}}{2}
$$

where $\Omega_{j, t}$ is the innovation covariance matrix and $\nu_{j, t}$ is the normalized innovation between the measurement $j$ and the predicted measurement of target $t$ :

$$
\nu_{j, t}^{2}=\tilde{\mathbf{z}}_{j, t}^{T} \Omega_{j, t}{ }^{-1} \tilde{\mathbf{z}}_{j, t}
$$

Thus the probability of an individual joint association event is given by

$$
P\left(\theta \mid \mathbf{Z}_{1: k}\right)=P_{D}^{N_{T}-n}\left(1-P_{D}\right)^{n} P_{F A}^{m_{k}-\left(N_{T}-n\right)} \prod_{\theta_{j, t} \in \theta} p_{j, t}
$$


Then the marginal association probability is given by

$$
\beta_{j, t}=\sum_{\theta: \theta_{j, t} \in \theta} P\left(\theta \mid \mathbf{Z}_{1: k}\right)
$$

The estimate of the $t^{t h}$ target is given by

$$
\begin{aligned}
\hat{\mathbf{x}}_{k \mid k, t} & =E\left[\mathbf{x}_{k, t} \mid \mathbf{Z}_{1: k}\right] \\
& =\sum_{j=1}^{m_{k}} E\left[\mathbf{x}_{k, t} \mid \theta_{j, t}, \mathbf{Z}_{1: k}\right] \beta_{j, t} \\
& =\sum_{j=1}^{m_{k}} \hat{\mathbf{x}}_{k \mid k, j, t} \beta_{j, t}
\end{aligned}
$$

where $\hat{\mathbf{x}}_{k \mid k, j, t}$ is the a posteriori state of target $t$ conditioned on the event $\theta_{j, t}$. 


\section{Chapter 4}

\section{Joint Detection And Tracking}

In this chapter, a novel algorithm is developed to explore the use of tracking information in detection (and vice versa) through joint detection and tracking. This enables us to jointly detect and track more than two unresolved targets using a monopulse radar with ideal single bin processing. This requires the transformation of the target state into detection parameter space, which involves high nonlinearities. The SMC method, which has proven to be effective in nonlinear non-Gaussian estimation problems, is used to handle the nonlinearities. In addition to the standard SMC steps, the hypothesis testing for detection is performed in each recursion, which is facilitated by likelihood computation through Monte Carlo integration of the particle filter. The formulation of the single recursion of the algorithm is given in the following section. It is also described with the help of a simple block diagram in the last section.

\subsection{Single Recursion of the Algorithm}

Consider a single recursion of the recursive Bayesian procedure of the SMC method for the closed-loop tracking of multiple closely spaced targets. At time $k-1$, the tracking information 
is represented by particle set $\left\{\begin{array}{cc}\mathbf{x}_{k-1}^{j, i} & w_{k-1}^{j, i}\end{array}\right\}_{j=1}^{N_{P}} N_{i=1}$, where $N_{T}$ is the number of tracks and $N_{P}$ is the number of particles per track. The particle state, $\mathbf{x}$, includes the target's kinematic state and the Average Radar Cross Section (ARCS) of the target. According to the Sampling Important Resampling (SIR) particle filter, the particles are predicted as

$$
\mathbf{x}_{k}^{j, i} \sim f_{k \mid k-1}\left(\mathbf{x}_{k}^{i} \mid \mathbf{x}_{k-1}^{j, i}\right) \quad j=1, \ldots, N_{p} ; \quad i=1, \ldots, N_{T}
$$

where $f_{k \mid k-1}(\cdot \mid \cdot)$ is the state transition model.

The radar resource managing routine determines the pointing direction for the next dwell, $\theta_{k}$, by considering these predicted positions $\left\{\begin{array}{ll}\mathbf{x}_{k}^{j, i} & w_{k \mid k-1}^{j, i}\end{array}\right\}_{j=1 \quad i=1}^{N_{P} N_{T}}$ where $w_{k \mid k-1}^{j, i}=w_{k-1}^{j, i}$. The signal parameters, $\Phi_{k}^{j, i}=\left[\begin{array}{lll}\alpha_{0 j i} & \eta_{b j i} & \eta_{e j i}\end{array}\right]^{T}$, corresponding to each particle is evaluated by using monopulse radar model from [12], [30].

$$
\Phi_{k}^{j, i} \sim g\left(\mathbf{x}_{k}^{j, i}, \theta_{k}\right) \quad j=1, \ldots, N_{p} ; \quad i=1, \ldots, N_{T}
$$

where $\alpha_{0 j i}$ is the average signal amplitude, $\eta_{b j i}$ is the bearing DOA and $\eta_{e j i}$ is the elevation DOA of the $j^{\text {th }}$ particle of target $i$.

The observation set, $\mathbf{Y}_{k}=\left\{\mathbf{y}_{k}^{m}\right\}_{m=1}^{M_{k}}$, is received for the dwell on the pointing direction $\theta_{k}$, where $M_{k}$ is the number of detected cells after thresholding, $\left.\mathbf{y}_{k}^{m}=\left[\begin{array}{cc}r_{k}^{m} & {\left[\mathbf{z}_{k}^{m}\right.}\end{array}\right]^{T}\right]^{T}, r_{k}^{m}$ is the $m^{t h}$ detected range bin centroid and $\mathbf{z}_{k}^{m}$ is the matched filter output of the $m^{\text {th }}$ detection. Assume that, after gating, $N_{k}$ tracks are corresponding to these detections. The validation matrix is constructed according to this gating [3], and different hypotheses are constructed for all possible events from the validation matrix. The hypothesis, $H_{h}$, is the one of the feasible events that are determined by the following assumptions of the ideal bin processing for unresolved targets:

- Each target falls into at most one cell 
- Each detection may be due to any number of targets

Assuming independent detections, the likelihood of the $h^{\text {th }}$ hypothesis, $H_{h}$, can be written as

$$
\Lambda\left(H_{h}\right)=p\left(\mathbf{Y}_{k} \mid H_{h}\right)=\prod_{m=1}^{M_{k}} p\left(\mathbf{y}_{k}^{m} \mid H_{h}\right)
$$

Using the total probability theorem, $p\left(\mathbf{y}_{k}^{m} \mid H_{h}\right)$ can be written as

$$
\begin{aligned}
p\left(\mathbf{y}_{k}^{m} \mid H_{h}\right) & =\int_{\boldsymbol{\Phi}^{N_{m, h}}} p\left(\mathbf{y}_{k}^{m} \mid \mathbf{\Phi}^{N_{m, h}}, H_{h}\right) p\left(\Phi^{N_{m, h}} \mid H_{h}\right) \quad d \Phi^{N_{m, h}} \\
& \left.=E_{p\left(\Phi^{N_{m, h}} \mid H_{h}\right.}\right) \quad\{\mu\}
\end{aligned}
$$

where $N_{m, h}$ is number of targets that cause the $m^{\text {th }}$ detection according to hypothesis $H_{h}$, and $E\{\cdot\}$ means the expectation operator. $\boldsymbol{\Phi}^{N_{m, h}}$ is the stacked parameter vector corresponding to the $N_{m, h}$ targets of detection $m$ given by

$$
\boldsymbol{\Phi}^{N_{m, h}}=\left[\begin{array}{lllll}
\left(\boldsymbol{\Phi}_{k}^{1}\right)^{T} & \ldots & \left(\boldsymbol{\Phi}_{k}^{i}\right)^{T} & \ldots & \left(\boldsymbol{\Phi}_{k}^{N_{m, h}}\right)^{T}
\end{array}\right]^{T}
$$

In the above, $\mu$ is the joint likelihood of the measurement given the stacked parameter vector $\boldsymbol{\Phi}^{N_{m, h}}$. That is,

$$
\begin{aligned}
\mu & =p\left(\mathbf{y}_{k}^{m} \mid \Phi^{N_{m, h}}, H_{h}\right) \\
& =p\left(r_{k}^{m} \mid \Phi^{N_{m, h}}, H_{h}\right) p\left(\mathbf{z}_{k}^{m} \mid \Phi^{N_{m, h}}, H_{h}\right) \\
& =p\left(r_{k}^{m} \mid X^{N_{m, h}}, H_{h}\right) p\left(\mathbf{z}_{k}^{m} \mid \Phi^{N_{m, h}}, H_{h}\right)
\end{aligned}
$$

with $X^{N_{m, h}}$ being the stacked state vector corresponding to $\Phi^{N_{m, h}}$. The first term in (4.93) is the probability of the event that $N_{m, h}$ targets fall into the $m^{\text {th }}$ cell and has uniform 
probability density function (PDF). The second term is the likelihood of signal $\mathbf{z}_{k}^{m}$ given the stacked parameter vector and has Gaussian PDF, which is given by (2.36).

Using Monte Carlo integration [8], (4.91) can be approximated as follows:

$$
p\left(\mathbf{y}_{k}^{m} \mid H_{h}\right) \approx \frac{1}{N_{c}} \sum_{j_{1}=1}^{N_{P}} \ldots \sum_{j_{i}=1}^{N_{P}} \ldots \sum_{j_{N_{m}, h}=1}^{N_{P}} \mu_{s}
$$

where $s$ is the set of the pair of indices of targets and particles given by

$$
s=\left\{\left[1 j_{1}\right], \ldots,\left[i j_{i}\right], \ldots,\left[N_{m, h} j_{N_{m, h}}\right]\right\}
$$

and $\mu_{s}$ is the joint likelihood of the particle set corresponding to $s$ and evaluated using (4.93). There are $N_{c}=\left(N_{p}\right)^{N_{m, h}}$ possible combinations of particles and denote them by $S=\{s\}$. Particles are updated with marginal likelihood, $\lambda^{j, i}$, which is given by

$$
\lambda^{j, i}=\sum_{s \in S^{j, i}} \mu_{s} \quad \text { with } \quad S^{j, i}=\{s: s \in S ;[i j] \in s\}
$$

The updated weight is given by

$$
w_{k}^{j, i}=w_{k-1}^{j, i} \lambda^{j, i} \quad j=1, \ldots, N_{p} ; \quad i=1, \ldots, N_{T}
$$

The hypothesis testing yields the correct detection event, i.e.,

$$
\hat{H}=\arg \max _{H_{h}} \Lambda\left(H_{h}\right)
$$

The corresponding updated particle set is taken as the correct representation of the posterior states of the targets at time $k$ and that completes a single recursion. 


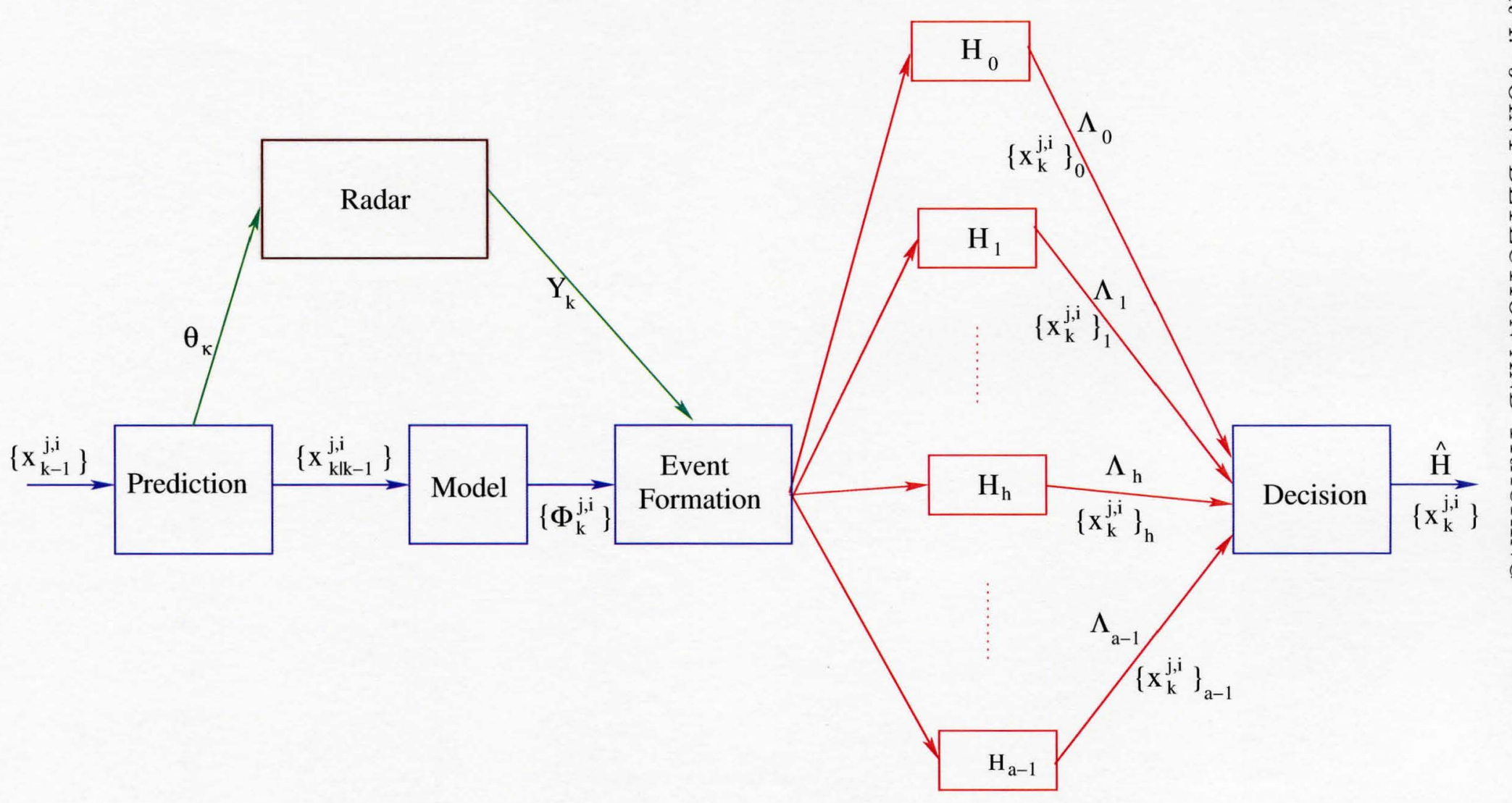

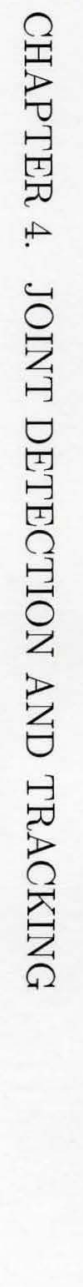

Figure 4.4: Joint detection and tracking 


\subsection{Block Diagram for Recursion}

Figure 4.4 depicts a simple block diagram of the algorithm and its key steps. The key steps are

- Predicted states are used to evaluate the detection parameters.

- They are also used to determine the pointing direction of the next dwell.

- Measurements are received for the dwell on the pointing direction.

- With the availability of measurements and predicted tracks, all possible events are formed.

- Likelihoods of all hypotheses are computed using Monte Carlo integration.

- The particle sets corresponding to the best hypothesis is taken as the correct representation of the target posterior. 


\section{Chapter 5}

\section{Posterior Covariance of Target State}

In this Chapter, the posterior covariance of the target state is derived to assess the performance bounds of the algorithm developed in Chapter 4. The recursive Riccati-like formula for the Fisher Information matrix for nonlinear state estimation from [27] is used to derive the posterior covariance of the target state. The inverse of the Fisher Information Matrix (FIM) is the Cramér-Rao Lower Bound (CRLB). The fundamental theory of behind the CRLB for parameter estimation can be found in [28] and the details of the Posterior Cramér-Rao Lower Bound (PCRLB) for state estimation can be found in [27]. The following section gives a brief background on CRLB and in the last section, the PCRLB of target states is derived.

\subsection{Background}

Let $Z$ represent a vector of measured data and $\hat{X}(Z)$ be an unbiased estimate of a parameter vector $X$. The Cramér-Rao lower bound for the estimation error covariance, $\mathrm{C}$, is defined to be the inverse of the Fisher Information Matrix, $J$, i.e.,

$$
C \triangleq \mathbb{E}\left\{[\hat{X}-X][\hat{X}-X]^{T}\right\} \geq J^{-1}
$$


where $J$ is defined in [28]. The inequality means that the difference $C-J^{-1}$ is a positive semi-definite matrix.

\subsection{Posterior Cramér-Rao Bounds for Target State Es- timation}

According to [27], the sequence of information matrices, $\left\{J_{k}^{i}\right\}$, for the state vector $\left\{\mathbf{x}_{k}^{i}\right\}$ of target $i$ obeys the recursion

$$
J_{k}^{i}=D_{k-1}^{22, i}-D_{k-1}^{21, i}\left(J_{k-1}^{i}+D_{k-1}^{11, i}\right)^{-1} D_{k-1}^{12, i}
$$

where $J_{k}^{i}$ and $J_{k-1}^{i}$ are Fisher information matrices of target $i$ at time $k$ and $k-1$, respectively, and

$$
\begin{aligned}
D_{k-1}^{11, i} & =E\left\{-\Delta_{\mathbf{x}_{k-1}^{i}}^{\mathbf{x}_{k-1}^{i}} \log p\left(\mathbf{x}_{k}^{i} \mid \mathbf{x}_{k-1}^{i}\right)\right\} \\
& =F^{T} Q^{-1} F \\
D_{k-1}^{12, i} & =E\left\{-\Delta_{\mathbf{x}_{k-1}^{2}}^{\mathbf{x}_{k}^{i}} \log p\left(\mathbf{x}_{k}^{i} \mid \mathbf{x}_{k-1}^{i}\right)\right\} \\
& =-Q^{-1} F \\
D_{k-1}^{21, i} & =E\left\{-\Delta_{\mathbf{x}_{k}^{i}}^{\mathbf{x}_{k-1}^{i}} \log p\left(\mathbf{x}_{k}^{i} \mid \mathbf{x}_{k-1}^{i}\right)\right\} \\
& =-F^{T} Q^{-1} \\
D_{k-1}^{22, i} & =E\left\{-\Delta_{\mathbf{x}_{k}^{i}}^{\mathbf{x}_{k}^{i}} \log p\left(\mathbf{x}_{k}^{i} \mid \mathbf{x}_{k-1}^{i}\right)\right\}+E\left\{-\Delta_{\mathbf{x}_{k}^{i}}^{\mathbf{x}_{k}^{i}} \log p\left(\mathbf{y}_{k}^{m} \mid \mathbf{x}_{k}^{i}\right)\right\} \\
& =Q^{-1}+J_{\mathbf{y}}^{i}
\end{aligned}
$$

In the above, $\Delta$ is the Hessian operator, $F$ is the Jacobian of the nonlinear process function $f$, $Q$ is the covariance matrix of the addictive process noise and $J_{\mathbf{y}}^{i}=E\left\{-\Delta_{\mathbf{x}_{k}^{i}}^{\mathbf{x}_{k}^{i}} \log p\left(\mathbf{y}_{k}^{m} \mid \mathbf{x}_{k}^{i}\right)\right\}$. 
(5.99) can be simplified using the matrix inversion lemma in [4], and written as

$$
J_{k}^{i}=\left[Q+F\left(J_{k-1}^{i}\right)^{-1} F^{T}\right]^{-1}+J_{\mathbf{y}}^{i}
$$

where $J_{\mathbf{y}}^{i}$ is the information contribution from associated measurement $\mathbf{y}_{k}^{m}$. In addition,

$$
\begin{aligned}
J_{\mathbf{y}}^{i} & =E\left\{-\Delta_{\mathbf{x}_{k}^{i}}^{\mathbf{x}_{k}^{i}} \log p\left(\mathbf{y}_{k}^{m} \mid \mathbf{x}_{k}^{i}\right)\right\} \\
& =E\left\{-\Delta_{\mathbf{x}_{k}^{i}}^{\mathbf{x}_{i}^{i}} \log p\left(\mathbf{r}_{k}^{m} \mid \mathbf{x}_{k}^{i}\right)\right\}+E\left\{-\Delta_{\mathbf{x}_{k}^{i}}^{\mathbf{x}_{k}^{i}} \log p\left(\mathbf{z}_{k}^{m} \mid \mathbf{x}_{k}^{i}\right)\right\} \\
& =J_{r}^{i}+J_{\mathbf{z}}^{i}
\end{aligned}
$$

where

$$
\begin{aligned}
& J_{r}^{i}=E\left\{-\Delta_{\mathbf{x}_{k}^{i}}^{\mathbf{x}_{k}^{i}} \log p\left(\mathbf{r}_{k}^{m} \mid \mathbf{x}_{k}^{i}\right)\right\} \\
& J_{\mathbf{z}}^{i}=E\left\{-\Delta_{\mathbf{x}_{k}^{i}}^{\mathbf{x}_{k}^{i}} \log p\left(\mathbf{z}_{k}^{m} \mid \mathbf{x}_{k}^{i}\right)\right\}
\end{aligned}
$$

The information contribution from range measurement, $J_{r}^{i}$, can also be written as

$$
J_{r}^{i}=\left.E\left\{\left[\nabla_{\mathbf{x}_{k}^{i}} \log p\left(r_{k}^{m} \mid \mathbf{x}_{k}^{i}\right)\right]\left[\nabla_{\mathbf{x}_{k}^{i}} \log p\left(r_{k}^{m} \mid \mathbf{x}_{k}^{i}\right)\right]^{T}\right\}\right|_{\mathbf{x}_{k}^{i}=\mathbf{x}_{0 k}^{i}}
$$

The range measurement uncertainty is uniformly distributed within the range bin and approximated as Gaussian in this computation. Thus,

$$
p\left(r_{k}^{m} \mid \mathbf{x}_{k}^{i}\right)=\mathcal{N}\left(\hat{r}_{k}^{i} ; r_{k}^{m}, \sigma_{r}^{2}\right)
$$

where $\hat{r}_{k}^{i}$ is the predicted range of target $i$ that is equal to $\sqrt{\mathbf{x}_{k}^{i}(1)^{2}+\mathbf{x}_{k}^{i}(3)^{2}+\mathbf{x}_{k}^{i}(5)^{2}}, \sigma_{r}^{2}=$ 
$b^{2} / 12$ and $b$ is the range bin size. The gradient, $\nabla_{\mathbf{x}_{k}^{i}} \log p\left(r_{k}^{m} \mid \mathbf{x}_{k}^{i}\right)$, can be written as

$$
\begin{aligned}
\nabla_{\mathbf{x}_{k}^{i}} \log p\left(r_{k}^{m} \mid \mathbf{x}_{k}^{i}\right) & =\frac{\left(\hat{r}_{k}^{i}-r_{k}^{m}\right)}{\hat{r}_{k}^{i} \sigma_{r}^{2}}\left[\mathbf{x}_{k}^{i}(1) 0 \mathbf{x}_{k}^{i}(3) 0 \mathbf{x}_{k}^{i}(5) 0\right]^{T} \\
& =\nu
\end{aligned}
$$

Therefore,

$$
J_{r}^{i}=\left.E\left[\nu \nu^{T}\right]\right|_{\mathbf{x}_{k}^{i}=\mathbf{x}_{0 k}^{i}}
$$

If the $m^{\text {th }}$ detection is due to targets $i=1, \ldots, N_{m}$, the information available in monopulse signal $\mathbf{z}_{k}^{m}$ is shared by them. Therefore, this information $J_{\mathbf{z}}$ can be written in terms of the information contribution to each target of (5.107) as follows:

$$
\mathbf{x}_{k}=\left[\mathbf{x}_{k}^{1 T}, \ldots, \mathbf{x}_{k}^{i T}, \ldots, \mathbf{x}_{k}^{N_{m} T}\right]^{T}
$$

and this is corresponding to the stacked state vector $\mathbf{x}_{k}$, and can be written as

$$
J_{\mathbf{z}}=\operatorname{diag}\left(\left[J_{\mathbf{z}}^{1}, \ldots, J_{\mathbf{z}}^{i}, \ldots, J_{\mathbf{z}}^{N_{m}}\right]\right)
$$

The information matrix $J_{\mathbf{z}}$ can also be written as

$$
J_{\mathbf{z}}=\left.E\left\{\left[\nabla_{\mathbf{x}_{k}} \log p\left(\mathbf{z}_{k}^{m} \mid \mathbf{x}_{k}\right)\right]\left[\nabla_{\mathbf{x}_{k}} \log p\left(\mathbf{z}_{k}^{m} \mid \mathbf{x}_{k}\right)\right]^{T}\right\}\right|_{\mathbf{x}_{k}=\mathbf{x}_{0 k}}
$$

where the likelihood function, $p\left(\mathbf{z}_{k}^{m} \mid \mathbf{x}_{k}\right)$, is given by (2.36). Let us define vector $\mathbf{a}=$ $\left[\begin{array}{llllll}a_{11} & a_{22} & a_{33} & a_{12} & a_{23} & a_{13}\end{array}\right]^{T}$ which contains the distinct elements of $P_{1}$. Similarly, define $\mathbf{b}=\left[\begin{array}{llllll}b_{11} & b_{22} & b_{33} & b_{12} & b_{23} & b_{13}\end{array}\right]^{T}$, which contains the distinct elements of the inverse 
of $P_{1}$. The gradient $\nabla_{\mathbf{x}_{k}} \log p\left(\mathbf{z}_{k}^{m} \mid \mathbf{x}_{k}\right)$ can be written as

$$
\begin{aligned}
\nabla_{\mathbf{x}_{k}} \log p\left(\mathbf{z}_{k}^{m} \mid \mathbf{x}_{k}\right) & =-\frac{1}{2} \nabla_{\mathbf{x}_{k}}\left(\mathbf{z}_{k}^{m} P^{-1} \mathbf{z}_{k}^{m}\right)-\frac{1}{2} \nabla_{\mathbf{x}_{k}} \log |P| \\
& =-\frac{1}{2} \nabla_{\mathbf{x}_{k}}\left(\mathbf{z}_{I k}^{m} P_{1}^{-1} \mathbf{z}_{I k}^{m}\right)-\frac{1}{2} \nabla_{\mathbf{x}_{k}}\left(\mathbf{z}_{Q k}^{m} P_{1}^{-1} \mathbf{z}_{Q k}^{m}\right)-\nabla_{\mathbf{x}_{k}} \log \left|P_{1}\right| \\
& =-\frac{1}{2} \nabla_{\mathbf{x}_{k}}\left(\mathbf{l}_{I}+\mathbf{l}_{Q}\right)^{T} \mathbf{b}-\nabla_{\mathbf{x}_{k}} \log c
\end{aligned}
$$

where

$$
\begin{aligned}
& \mathbf{z}_{I k}^{m}=\left[\begin{array}{lll}
s_{I k} & d_{b I k} & d_{e I k}
\end{array}\right]^{T} \quad \text { (in phase part of the signal) } \\
& \mathbf{z}_{Q k}^{m}=\left[\begin{array}{lll}
s_{Q k} & d_{b Q k} & d_{e Q k}
\end{array}\right]^{T} \quad \text { (quadrature part of the signal) } \\
& \mathrm{l}_{I}=\left[\begin{array}{llllll}
s_{I k}{ }^{2} & d_{b I k}{ }^{2} & d_{e I k}{ }^{2} & 2 s_{I k} d_{b I k} & 2 d_{b I k} d_{e I k} & 2 s_{I k} d_{\text {eIk }}
\end{array}\right]^{T} \\
& \mathbf{l}_{Q}=\left[\begin{array}{llllll}
s_{Q k}{ }^{2} & d_{b Q k}{ }^{2} & d_{e Q k}{ }^{2} & 2 s_{Q k} d_{b Q k} & 2 d_{b Q k} d_{e Q k} & 2 s_{Q k} d_{e Q k}
\end{array}\right]^{T} \\
& c=\left|P_{1}\right|=a_{11} a_{22} a_{33}-a_{11} a_{23}^{2}-a_{12}^{2} a_{33}+2 a_{12} a_{13} a_{23}-a_{13}^{2} a_{22}
\end{aligned}
$$

It can be further simplified as

$$
\begin{aligned}
\nabla_{\mathbf{x}_{k}} \log p\left(\mathbf{z}_{k}^{m} \mid \mathbf{x}_{k}\right) & =-\left[\frac{1}{2}\left(\mathbf{l}_{I}+\mathbf{l}_{Q}\right)^{T} \frac{d}{d \mathbf{a}} \mathbf{b}+\left(\frac{d}{d \mathbf{a}} \log c\right)^{T}\right] \frac{d}{d \mathbf{x}_{k}} \mathbf{a} \\
& =v^{T}
\end{aligned}
$$


where the Jacobian $\frac{d}{d \mathbf{a}} \mathbf{b}$ is given by

$$
\frac{d}{d \mathbf{a}} \mathbf{b}=\frac{1}{c}\left[\begin{array}{cccccc}
-\frac{e_{1}^{2}}{c} & a_{33}-\frac{e_{1} e_{2}}{c} & a_{22}-\frac{e_{1} e_{3}}{c} & -\frac{e_{1} e_{4}}{c} & -2 a_{23}-\frac{e_{1} e_{5}}{c} & -\frac{e_{1} e_{6}}{c} \\
a_{33}-\frac{e_{1} e_{2}}{c} & -\frac{e_{2}^{2}}{c} & a_{11}-\frac{e_{2} e_{3}}{c} & -\frac{e_{2} e_{4}}{c} & -\frac{e_{2} e_{5}}{c} & -2 a_{13}-\frac{e_{2} e_{6}}{c} \\
a_{22}-\frac{e_{1} e_{3}}{c} & a_{11}-\frac{e_{2} e_{3}}{c} & -\frac{e_{3}^{2}}{c} & -2 a_{12}-\frac{e_{3} e_{4}}{c} & -\frac{e_{3} e_{5}}{c} & -\frac{e_{3} e_{6}}{c} \\
\frac{e_{1} e_{7}}{c} & \frac{e_{2} e_{7}}{c} & -a_{12}+\frac{e_{3} e_{7}}{c} & -a_{33}+\frac{e_{4} e_{7}}{c} & a_{13}+\frac{e_{5} e_{7}}{c} & a_{23}+\frac{e_{6} e_{7}}{c} \\
-a_{23}+\frac{e_{1} e_{8}}{c} & \frac{e_{2} e_{8}}{c} & \frac{e_{3} e_{8}}{c} & a_{13}+\frac{e_{4} e_{8}}{c} & -a_{11}+\frac{e_{5} e_{8}}{c} & a_{12}+\frac{e_{6} e_{8}}{c} \\
\frac{e_{1} e_{9}}{c} & -a_{13}+\frac{e_{2} e_{9}}{c} & \frac{e_{3} e_{9}}{c} & a_{23}+\frac{e_{4} e_{9}}{c} & a_{12}+\frac{e_{5} e_{9}}{c} & -a_{22}+\frac{e_{6} e_{9}}{c}
\end{array}\right]
$$

The gradient $\frac{d}{d \mathbf{a}} \log c$ is given by

$$
\frac{d}{d \mathbf{a}} \log c=\frac{1}{c}\left[\begin{array}{llllll}
e_{1} & e_{2} & e_{3} & e_{4} & e_{5} & e_{6}
\end{array}\right]^{T}
$$

with

$$
\begin{aligned}
& e_{1}=a_{22} a_{33}-a_{23}^{2} \\
& e_{2}=a_{11} a_{33}-a_{13}^{2} \\
& e_{3}=a_{11} a_{22}-a_{12}^{2} \\
& e_{4}=2\left(a_{13} a_{23}-a_{12} a_{33}\right) \\
& e_{5}=2\left(a_{12} a_{13}-a_{11} a_{23}\right) \\
& e_{6}=2\left(a_{12} a_{23}-a_{13} a_{22}\right) \\
& e_{7}=a_{12} a_{33}-a_{13} a_{23} \\
& e_{8}=a_{11} a_{23}-a_{12} a_{13} \\
& e_{9}=a_{13} a_{22}-a_{12} a_{23}
\end{aligned}
$$


and the Jacobian $\frac{d}{d \mathbf{x}_{k}} \mathbf{a}$ is given by

$$
\frac{d}{d \mathbf{x}_{k}} \mathbf{a}=\left[\begin{array}{lllll}
A_{1} & \ldots & A_{i} & \ldots & A_{N_{m}}
\end{array}\right]
$$

with

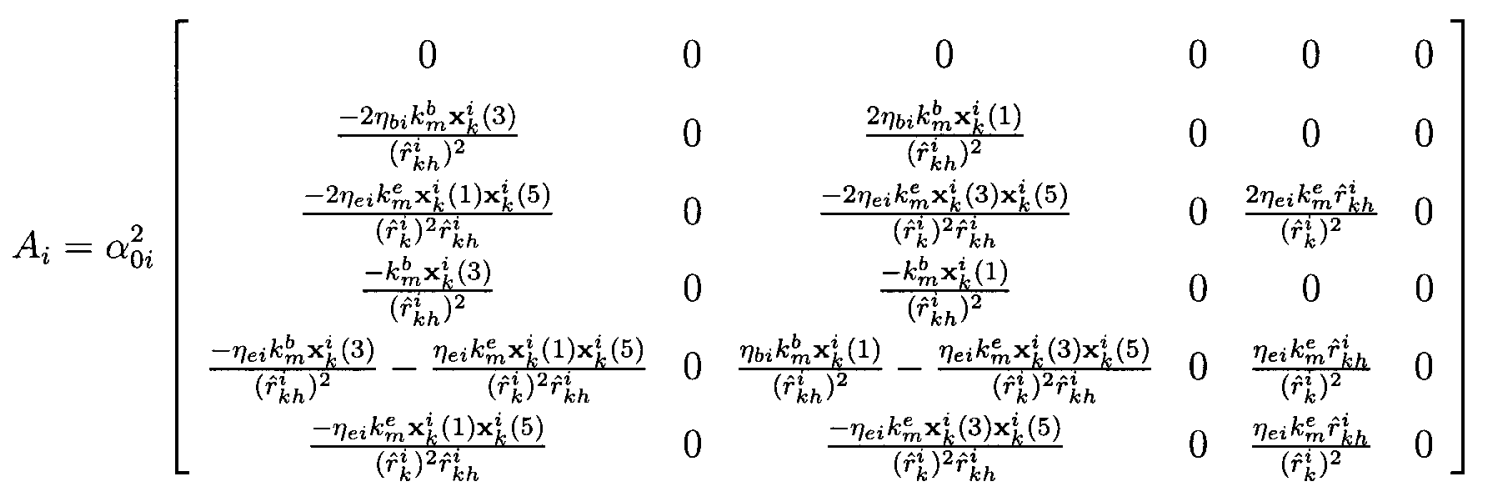

In the above, $\hat{r}_{k h}^{i}$ is the azimuth range given by $\sqrt{\mathbf{x}_{k}^{i}(1)^{2}+\mathbf{x}_{k}^{i}(3)^{2}}$. In the above differentiation, a linearly approximated monopulse error function (2.33) is used. Therefore, the information contributed by monopulse signal measurement is

$$
J_{\mathbf{z}}=\left.E\left\{v v^{T}\right\}\right|_{\mathbf{x}_{k}=\mathbf{x}_{0 k}}
$$

This expectation can be computed using Monte Carlo integration. 


\section{Chapter 6}

\section{Simulation Studies}

In this chapter, results of the simulation studies for the algorithm that was developed in Chapter 4 are presented. There are two objectives for our simulation studies: the first is to evaluate to the performance in terms of track purity and the second is to analyze its tracking error performance relative to the PCRLB derived in Chapter 5. The first scenario used in the simulations is shown in Figure 6.5, where three aircrafts that are initially resolved and travel such that they approach each other with linear motion for $20 s$ and then make a coordinated-turn for $20 s$ so that they are brought into a convoy formation. During this period, the targets start to become unresolved. After $5 s$ of convoy movement, during which the targets are fully unresolved, they start to separate with $20 \quad s$ of coordinated-turn followed by linear motion for $20 \mathrm{~s}$. During the convey movements targets move with a constant velocity of $100 \mathrm{~m} / \mathrm{s}$ in parallel courses separated by the distance of $0.4 \mathrm{~km}$. The ARCS of each target is $1.4 m^{2}$.

The radar is located at the origin of the coordinates. The radar is a nominal $4 \mathrm{GHz}$ phased array using amplitude comparison monopulse with uniform illumination across the array. A waveform of four subpulses (frequency diversity) is used. In this simulation a 
discretized white noise acceleration model [4] for target's kinematic state transition model and a white noise process model for ARCS transition model are used for state transition model in (4.89). That is,

$$
\begin{aligned}
\xi_{k \mid k-1} & =F \xi_{k-1 \mid k-1}+\Gamma v \\
\sigma_{k \mid k-1} & =\sigma_{k-1 \mid k-1}+n
\end{aligned}
$$

where $\xi$ is the target kinematic state, $\sigma$ is the target ARCS, $v$ is a Gaussian noise vector realization with independent components having a standard deviation of $10 \mathrm{~m} / \mathrm{s}^{2}$, each $n$ is a white noise with standard deviation $0.05 \mathrm{~m}^{2}$ and the matrices $F$ and $\Gamma$ are given by

$$
\begin{aligned}
F & =\left[\begin{array}{cccccc}
1 & \delta & 0 & 0 & 0 & 0 \\
0 & 1 & 0 & 0 & 0 & 0 \\
0 & 0 & 1 & \delta & 0 & 0 \\
0 & 0 & 0 & 1 & 0 & 0 \\
0 & 0 & 0 & 0 & 1 & \delta \\
0 & 0 & 0 & 0 & 0 & 1
\end{array}\right] \\
\Gamma & =\left[\begin{array}{ccc}
\delta^{2} / 2 & 0 & 0 \\
\delta & 0 & 0 \\
0 & \delta^{2} / 2 & 0 \\
0 & \delta & 0 \\
0 & 0 & \delta^{2} / 2 \\
0 & 0 & \delta
\end{array}\right]
\end{aligned}
$$

where $\delta$ is the time duration between the two consecutive dwells. 
The results from 50 Monte Carlo runs are presented below. Figure 6.6 shows the 50 Monte Carlo run average. The average tracks are almost following the truth with degraded performance during target maneuvering intervals. The estimated tracks of 50 Monte Carlo runs are overlayed in Figure 6.7, which demonstrates track purity. Figure 6.8 shows the average position Root Mean Squared Error (RMSE). It shows how the uncertainty increases with the number of targets and reduces once the targets are resolved again. 


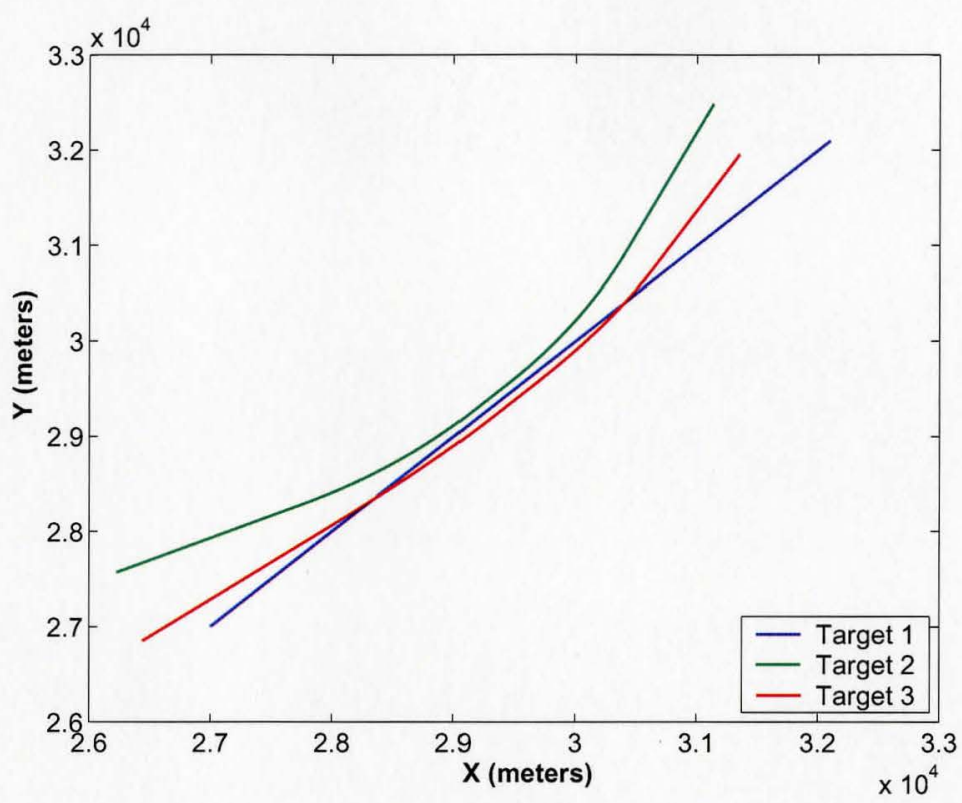

(a) XY Plane

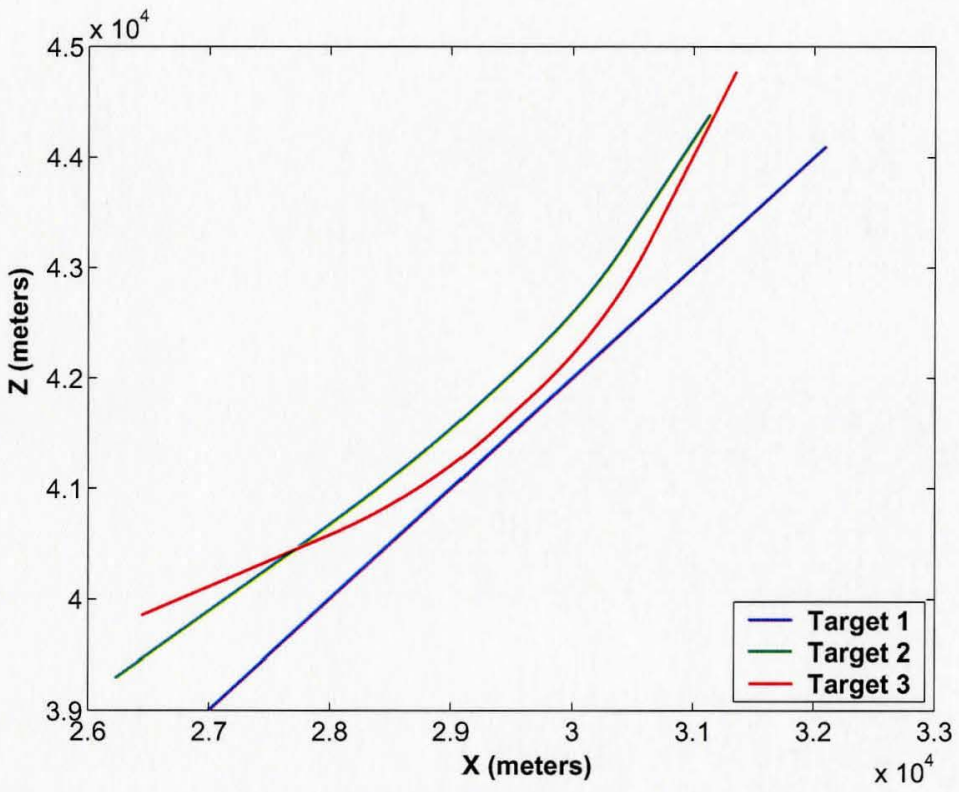

(b) XZ Plane

Figure 6.5: Target trajectories of the first scenario 


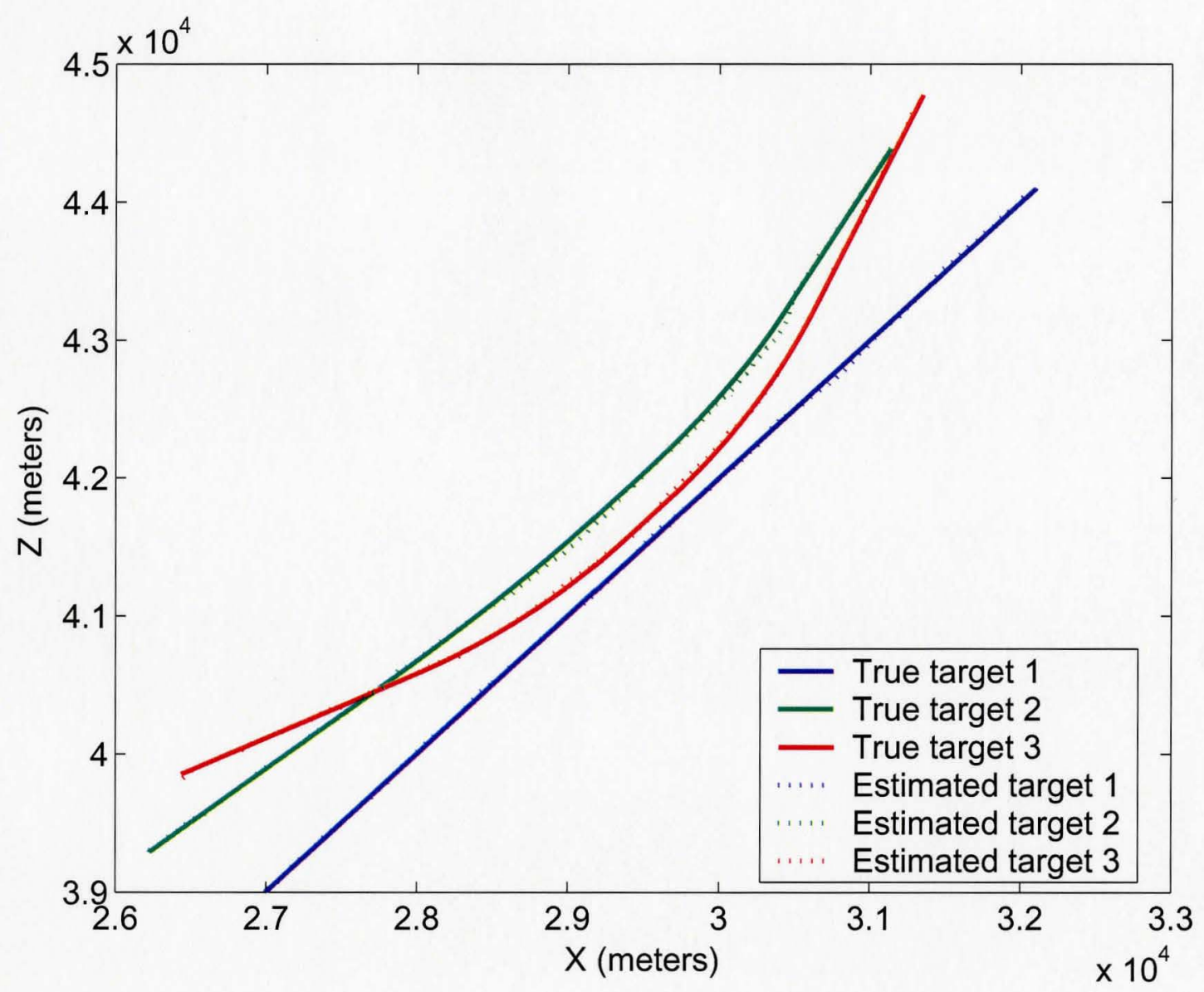

Figure 6.6: Tracks from 50 Monte Carlo runs (average tracks) 


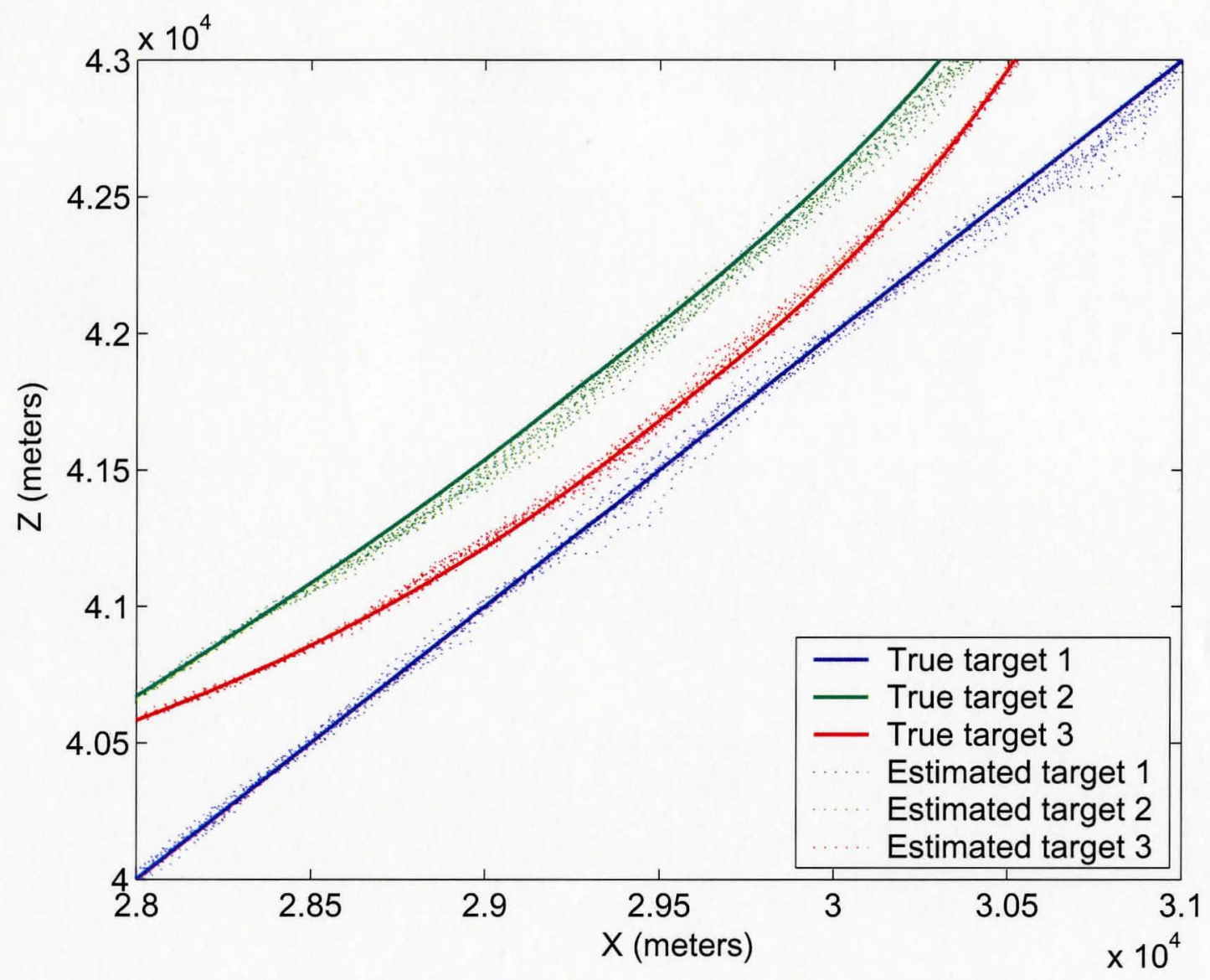

Figure 6.7: Tracks from 50 Monte Carlo runs (overlayed) 


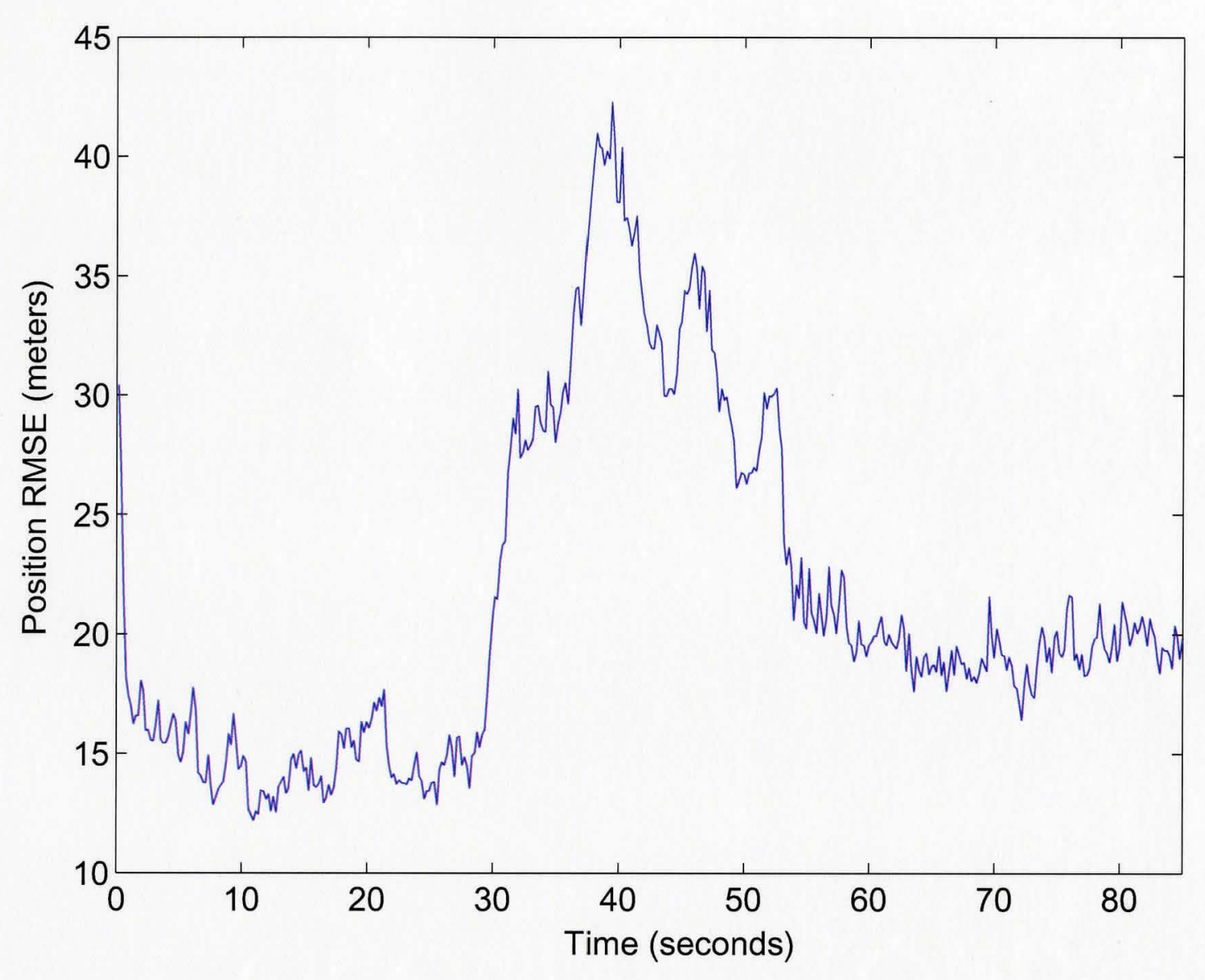

Figure 6.8: Average position RMSE 
In the second part of this study, a scenario set similar to the eighth set of target trajectories in [30], which consists of a number of inbound aircraft flying along parallel courses, is used with the same radar setup as in the first simulation study. The aircraft have an initial range of $70 \mathrm{~km}$ and maintain a constant velocity of $200 \mathrm{~m} / \mathrm{s}$ with a separation of $0.4 \mathrm{~km}$ so that they are unresolved during the whole period considered in the simulation. The ARCS for all targets is $1.4 \mathrm{~m}^{2}$. The trajectories for the scenario with three targets is shown in Figure 6.9.

Figure 6.10 shows the estimated position RMSE and the corresponding lower bounds for the single target case. As expected, the algorithm performs well, matching the lower bounds. For the case of two targets, as shown in Figure 6.11, it is approaching the lower bound and agrees with results of [23]. Similarly, it is somewhat approaching for the case of three unresolved targets as shown in Figure 6.12. This is a clear demonstration of the possible detection of more than two targets with the help of tracking information. 


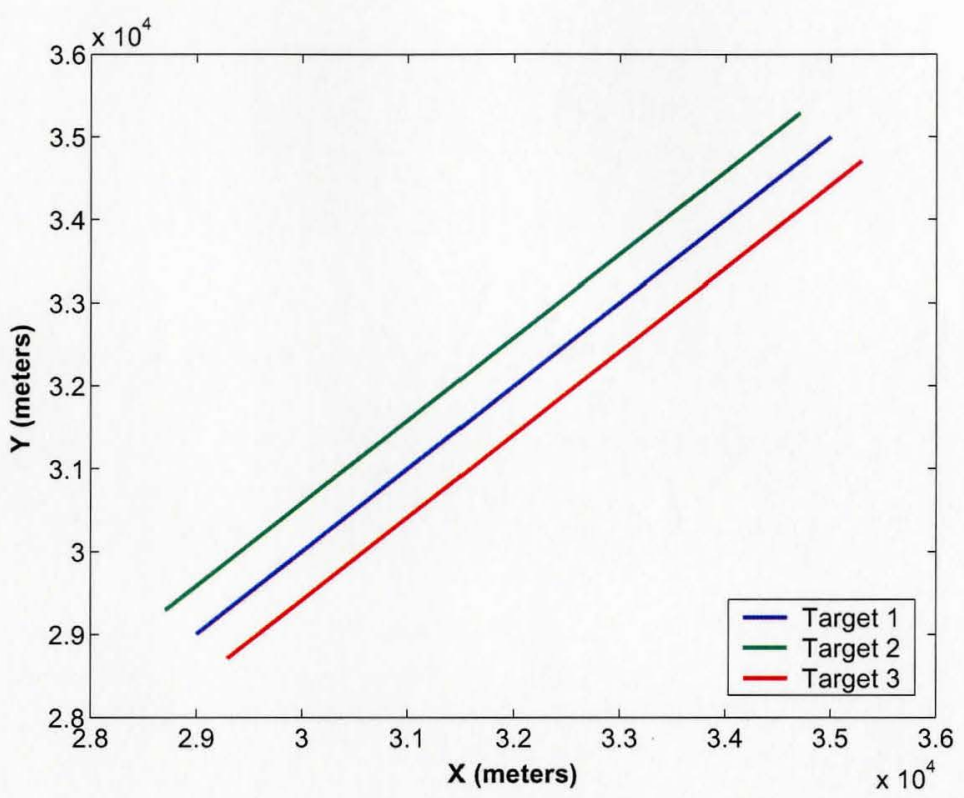

(a) XY Plane

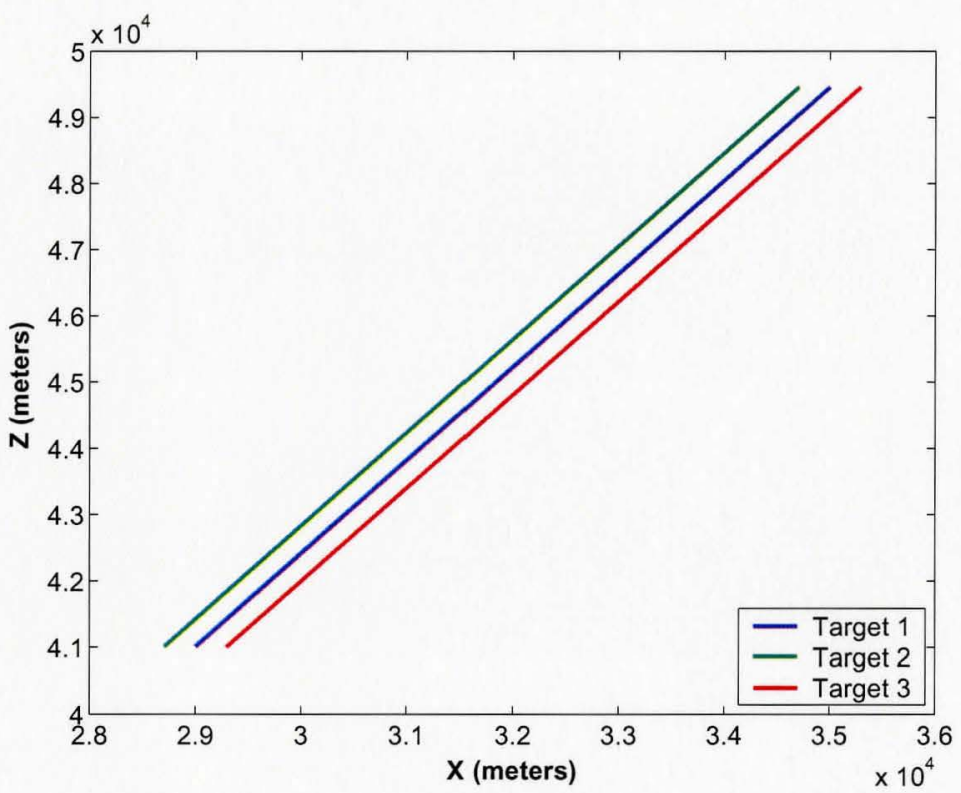

(b) XZ Plane

Figure 6.9: Target trajectories of the second scenario set with three targets 


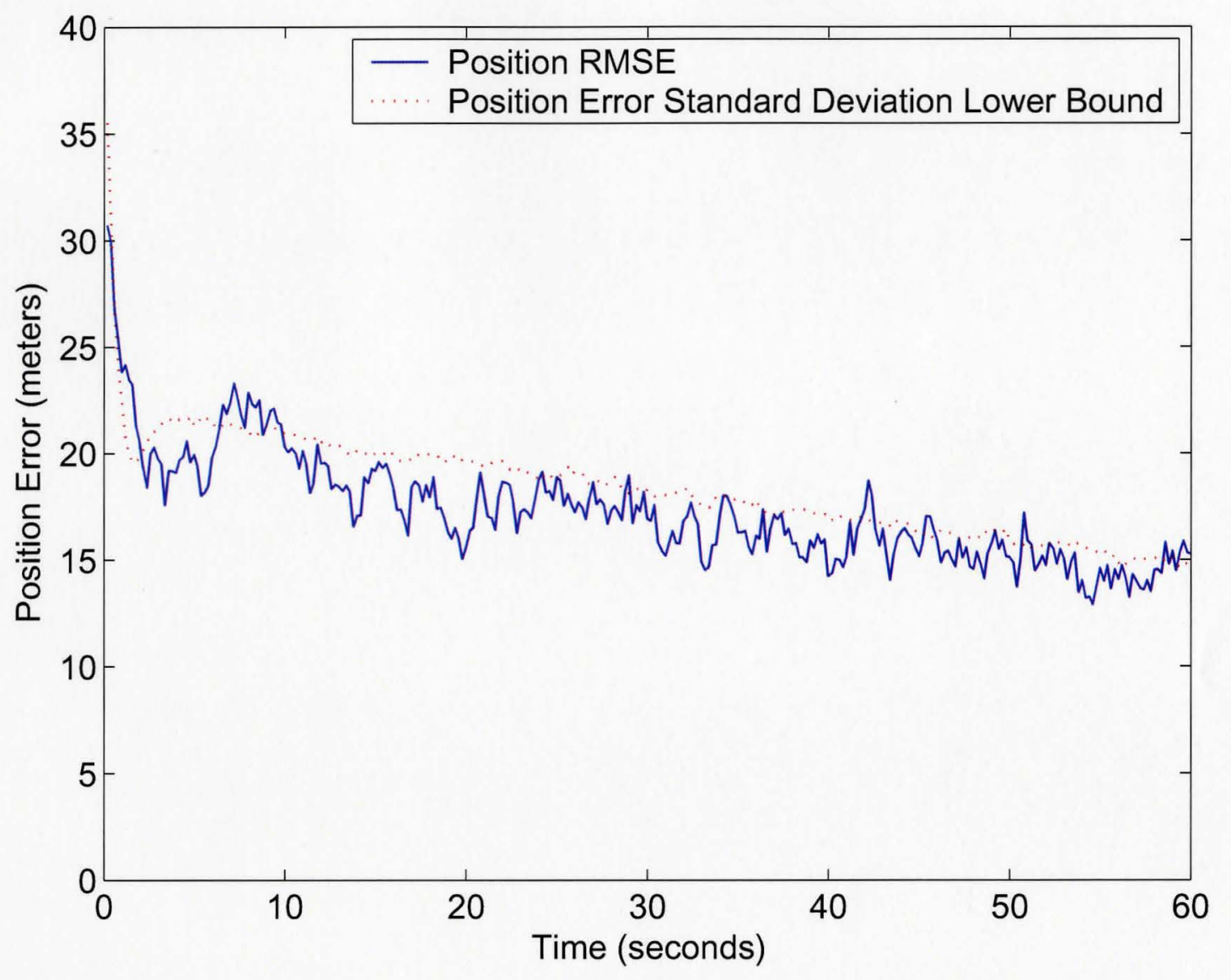

Figure 6.10: Average position RMSE and posterior position standard deviation lower bound for a single target 


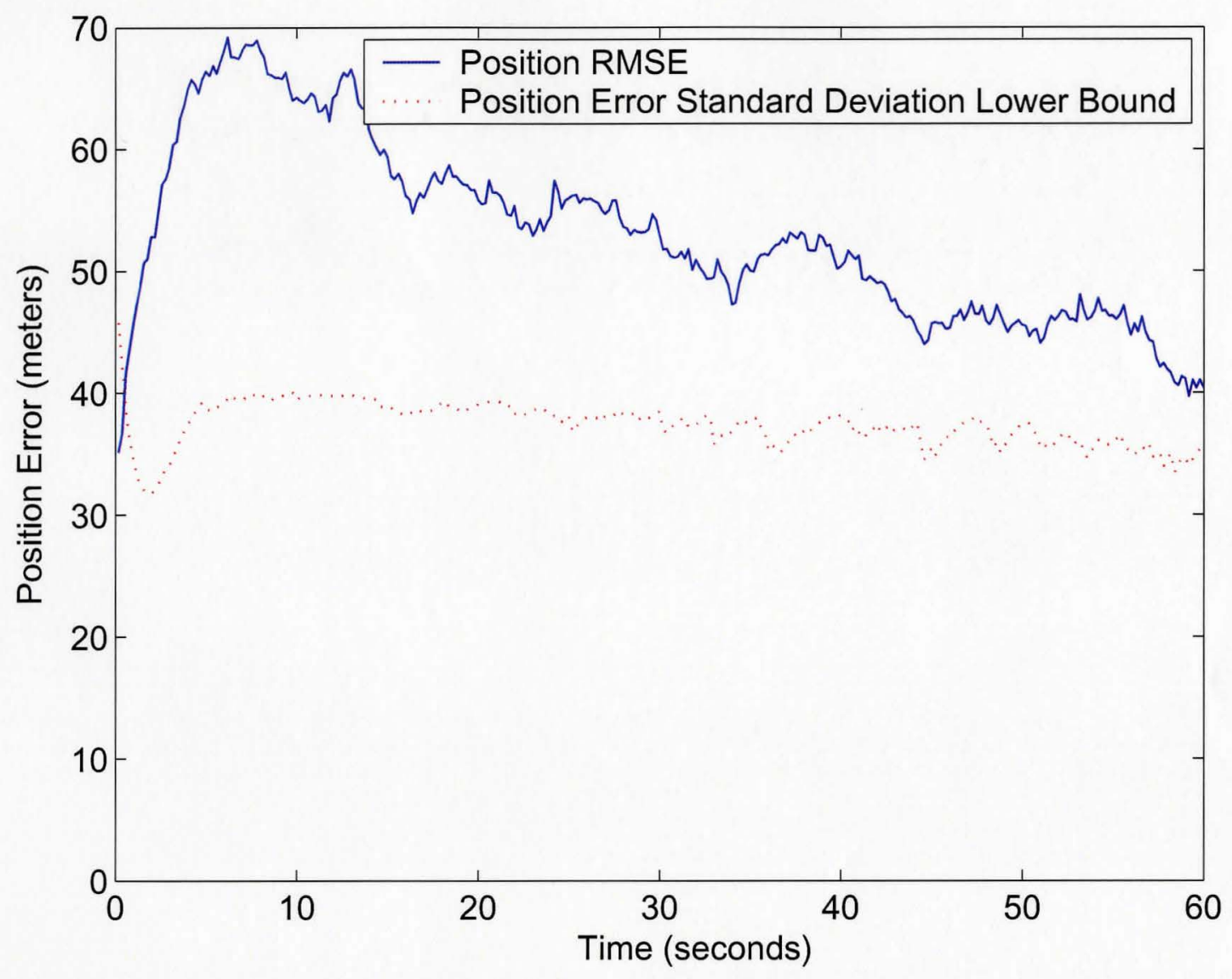

Figure 6.11: Average position RMSE and posterior position standard deviation lower bound for two unresolved targets 


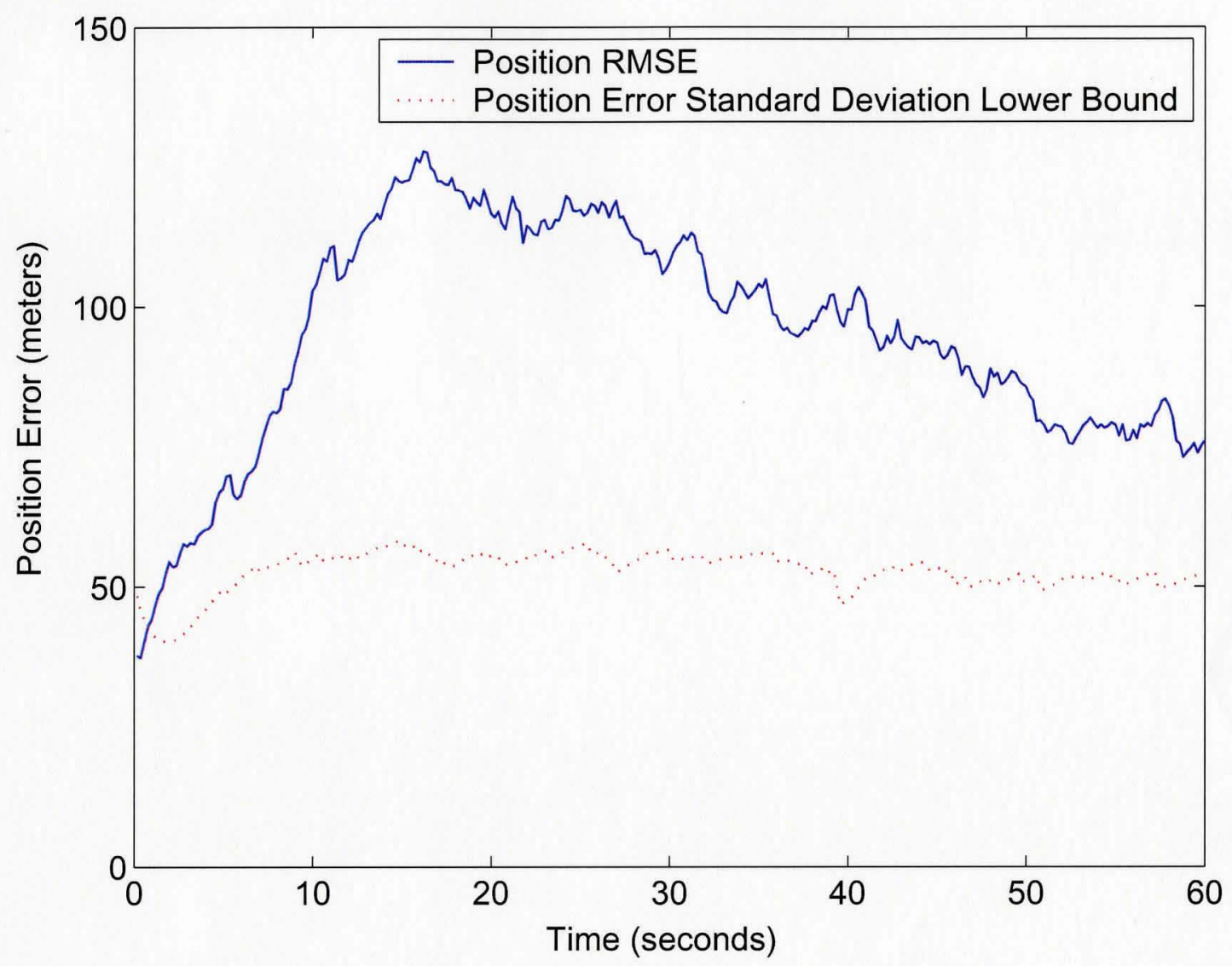

Figure 6.12: Average position RMSE and posterior position standard deviation lower bound for three unresolved targets 


\section{Chapter 7}

\section{SUMMARY}

\subsection{Conclusions}

An algorithm is developed to jointly detect and track unresolved targets with a monopulse radar using ideal bin processing. It explores the use of tracking information in detection. For this purpose, it constructs the hypotheses for the possible detection events and selects the one with the highest score via hypothesis testing. The particle filtering method makes it possible to evaluate the likelihoods of the hypotheses through Monte Carlo integration. The particle set corresponding to the best hypothesis represents the target posterior. Posterior covariance is derived to assess the algorithm using a recursive formula for the Fisher Information Matrix. Simulation studies confirm the possible joint extraction and tracking of more than two targets using single bin processing. 


\subsection{Recommendations for Future Work}

In this study, we considered ideal single bin processing, where target returns are assumed to be at only one matched filter sampling point. But, in real-world problems, target returns are spread over more than one sample point. The joint bin processing for this target spill-over into adjacent bin has proven to improve the detection $[31,32]$. Keeping this in mind, current work can be extended to exploit the target spill-over so that the estimation accuracy could be improved. The second possible extension of this work is to accommodate automatic track initialization and termination. In the current implementation, only the maintenance of the tracks corresponding to multiple unresolved targets is considered. With suitable birth and death models, the current work can be extended to handle more realistic problem where targets appear and disappear intermittently. 


\section{Bibliography}

[1] M. S. Arulampalam and B. Ristic, (2000) "Comparison of the particle filter with rangeparameterised and modified polar EKFs for angle-only tracking", Proceedings of SPIE on Signal and Data Processing of Small Targets, Vol. 4048, pp. 288-299, Orlando, FL, Apr 2000.

[2] Arulampalam, M.S., Maskell, S., Gordon, N., and Clapp, T., (2002) A Tutorial on Particle Filters for Online Nonlinear/Non-Gaussian Bayesian Tracking, IEEE Transactions on Signal Processing, Vol. 50, No. 2, pp. 174 - 188, Feb. 2002.

[3] Bar-Shalom, Y., and Li, X. R. (1995)Multitarget-Multisensor Tracking: Principles and Techniques. Stprrs, CT: YBS Publishing, 1995.

[4] Bar-Shalom,Y., Li, X., and Kirubarajan, T. (1999) Estimation and Tracking: Principles Techniques and Software. Storrs, CT: YBS publishers, 1999.

[5] Blair, W. D., and Brandt-Pearce, M. (1998) Unresolved Rayleigh target detection using Monopulse measurements, IEEE Transactions on Aerospace and Electronic Systems, Vol. 34, No. 2, pp. 543 - 552, Apr. 1998.

[6] Blair, W. D., and Brandt-Pearce, M. (1998) Statistical Description of monopulse Parameters for Tracking Rayleigh Targets, IEEE Transactions on Aerospace and Electronic Systems, Vol. 34, No. 2, pp. 597 - 611, Apr. 1998. 
[7] Blair, W. D., and Brandt-Pearce, M. (2001) Monopulse DOA Estimation of Two Unresolved Rayleigh Targets, IEEE Transactions on Aerospace and Electronic Systems, Vol. 37, No. 2, pp. $542-469$, Apr. 2001.

[8] Doucet, A., De Freitas, F. G., and Gordon, N. J., Eds., Sequential Monte Carlo Methods in Practice. New York: Springer-Verlag, 2001.

[9] N. J. Gordon, D. J. Salmond and A. F. M Smith, "Novel approach to nonlinear/nonGaussian Bayesian state estimation", IEE Proceedings F, Vol. 140, No. 2, pp .107-113, Apr 1993.

[10] Haykin, S.,Litva, J., and Shepherd, T.(1993)Radar Array Processing. New York: Springer-Verlag, 1993.

[11] S. Julier,(1998) "A skewed approach to filtering", Proceedings of SPIE on Signal and Data Processing of Small Targets, Vol. 3373, pp. 271-282, Orlando, FL, Apr 1998.

[12] Kirubarajan, T., Bar-Shalom, Y., Blair, W. D., and Watson, G. A. (1998) IMMPDAF for radar management and tracking benchmark with ECM. IEEE Transactions on Aerospace and Electronic Systems, Vol 34, No. 4 ,pp. 1115 - 1132, Oct. 1998.

[13] Ksienski, A. A., and McGhee, R. B. (1968) A decision theoretic approach to the angular resolution and parameter estimation problem for multiple targets, IEEE Transactions on Aerospace and Electronic Systems, AES-4 pp. 443 - 455, May 1968.

[14] J. MacCormick and A. Blake, "Probabilistic exclusion principle for tracking multiple objects", Proceedings of the IEEE International Conference on Computer Vision, Vol 1, pp. 572-578, Kerkyra, Greece, Sep 1999. 
[15] Nickel, U. (1993) Monopulse estimation with adaptive arrays. IEE Proceedings, Pt. F, 140, 5, pp. $303-308,1993$.

[16] Papoulis, A., and Pillai, S. U., Probability, Random Variables and Stochastic Processes, McGraw - Hill, 2002.

[17] Peebles, P. Z., and Berkowitz, R. S. (1968) Multiple-target monopulse radar processing techniques. IEEE Transaction on Aerospace and Electronic Systems, AES-4 pp. $845-$ 854, Nov. 1968.

[18] Pollon, G. E., and Lank, G. W. (1968) Angular tracking of two closely spaced radar targets, IEEE Transaction onAerospace and Electromic Systems, AES-4 pp. 541 - 550, July 1968.

[19] Rhodes, D. R., (1959) Introduction to Monopulse, McGraw-Hill,1959.

[20] Ristic, B., Arulampalam, S., and Gordon, N., Beyond the Klaman Filter: Particle Filters for Tracking Applications, Artech House, 2004.

[21] D. B. Rubin, "Using the SIR algorithm to simulate posterior distributions", Bayesian Statistics, 3, Oxford University Press, 1992.

[22] Sherman, S. (1984) Monopulse Principles and Techniques. Boston: Artech House, 1984.

[23] Sinha, A., Kirubarajan, T., and Bar-Shalom, Y. (2002) Maximum likelihood angle extractor for two closely spaced targets. IEEE Transactions on Aerospace and Electronic Systems, Vol. 38, No. 2. pp. 183 - 203. Jan. 2002.

[24] Sinha, A., Bar-Shalom, Y., Blair, W. D., and Kirubarajan, T. (2003) Radar measurement Extraction in the Presence of Sea-Surface Multipath. IEEE Transactions on Aerospace and Electronic Systems, Vol. 39, No. 2. pp. 550 - 567. Apr. 2003. 
[25] Skolnik, M. I. (1980), Introduction to Radar Systems, McGraw-Hill, 1980.

[26] A. F. M. Smith and A. E. Gelfand, "Bayesian statistics without tears: A sampling resampling perspective", The American Statistician, Vol. 46 No. 2, pp. 84-88, 1992.

[27] Tichavský, P., Muravchik, C.H., and Nehorai, A. (1998) Posterior Cramér - Rao Bounds for Discrete - time Nonlinear Filtering. IEEE Transactions on Signal Processing, Vol. 46, No. 5. pp. 1386 - 1396. May 1998.

[28] H. Van Trees, Detection, Estimation and Modulation Theory, Part I, Wiley, New York, 2001.

[29] Wang, Z., Sinha, A., Willett, P., and Bar-Shalom, Y. (2004) Angle Estimation for Two Unresolved Targets with Monopulse Radar. IEEE Transactions on Aerospace and Electronic Systems, Vol. 40, No. 3. pp. 998 - 1019. July 2004.

[30] Watson, G. A., and McCabe, D. H. (1999) Benchmark problem with a multisensor framework for radar resource allocation and tracking of highly maneuvering targets, closely-spaced targets, and targets in the presentce of sea-surface-induced multipath. Technical Report NSWCDD, Dahlgren, VA, Mar. 1999.

[31] Zhang, X., Willett, P., and Bar-Shalom, Y. (2003)Detection and Localization of Multiple Unresloved Targets via Joint Multiple-Bin Processing. Proceedings of IEEE Radar conference, Huntsville AL,May 2003.

[32] Zhang, X., Willett, P., and Bar-Shalom, Y. (2005) Monopulse Radar Detection and Localization of Multiple Unresloved Targets via Joint Bin Processing. IEEE Transactions on Signal Processing, Vol. 53, No. 4,pp. 1225 - 1236. Apr. 2005. 